\title{
Transfer of calibration between hand and foot: Functional equivalence and fractal fluctuations
}

\author{
Damian G. Stephen • Alen Hajnal
}

Published online: 20 May 2011

(C) Psychonomic Society, Inc. 2011

\begin{abstract}
Transfer of competency in a perceptual task often depends on shared information between anatomically different perceptual subsystems. The problem of studying transfer involves isolating conditions of similarity and then trying to account for any resulting differences in transfer. To respect this twofold aspect, this article takes a twopronged approach to transfer in dynamic touch. The present research first tests the hypothesis that functional equivalence supports the transfer of dynamic touch. Participants were trained to wield unseen objects with the hand or foot and were then tested on anatomically disparate limbs (i.e., the foot or hand, respectively). Next, we examined motion capture of these wielding behaviors for predictors of any asymmetry in transfer. Temporally fractal fluctuations of exploratory behavior can modulate information detection, and we tested whether the fractality of wielding might predict asymmetries in transfer across disparate limbs. Results suggest that transfer of training to anatomically disparate limbs respects functional conditions of similarity and also that the degree of temporally fractal fluctuations predicted limb differences in transfer.
\end{abstract}

D. G. Stephen $(\bowtie)$

Wyss Institute for Biologically Inspired Engineering,

Harvard University,

3 Blackfan Circle, Floor 5,

Boston, MA 02115, USA

e-mail: damian.stephen@wyss.harvard.edu

D. G. Stephen

Department of Psychiatry, Children's Hospital Boston,

Harvard Medical School,

Boston, MA, USA

A. Hajnal

Department of Psychology, University of Southern Mississippi,

Hattiesburg, MS, USA
Keywords Dynamic touch · Transfer - Calibration - Foot . Hand · Feedback

\section{Transfer of calibration between hand and foot}

An essential aspect of the organization of the perceptual system is the transfer of competency. For example, will practice kicking a ball with the foot have any benefit for how one throws a ball with the hand? The question is whether one perceptual subsystem (i.e., for kicking) can influence another (i.e., for throwing). Transfer of competency provides insights into the organization of the perceptual system at large, because it reveals common ground among perceptual subsystems. Perception depends on the detection of information useful for action, but transfer suggests that information detected with one part of the body can be shared with another part of the body. The question behind transfer of competency leads quickly to a twofold challenge. First, transfer requires conditions of similarity across perceptual subsystems, and second, the appearance of transfer is subject to anatomical differences in capacity for detecting information.

Past research has suggested that transfer can be supported by anatomical similarity and functional equivalence. In the former case, transfer may depend on similar anatomical components having similar capacities for detecting information, and in the latter case, transfer may depend on the similarity of function regardless of different anatomical capacities for detecting information. Functional equivalence highlights the intriguing notion that information for perception may be deeply grounded in the actions that it serves (e.g., Arzamarski, Isenhower, Kay, Turvey, \& Michaels 2010; Gibson, 1979). Functional equivalence poses a particularly interesting case because it also forces the question of how 
anatomical differences might be characterized and reconciled to permit transfer of competency. Capacities for detecting information will vary with the exploratory modes available to any anatomical component (Galloway \& Thelen, 2004; van de Langenberg, Kingma, \& Beek, 2006). Hence, the efficacy of transfer from one anatomical component to another may depend on the exploratory movements by each anatomical component involved. However, exploratory movements must be able to share detected information across some common ground between anatomically disparate parts.

The purpose of this article is to elucidate the dynamics of transfer in the context of functional equivalence across anatomically disparate components. In order to do justice to these aforementioned concerns, the article will address a single experiment in two parts. In the first part, we will demonstrate functional equivalence in performance for a given perceptual skill across anatomical disparities and across different sets of stimuli. This first part will be restricted to the behavioral data, testing whether training of one anatomical component leads to recalibration of perceptual judgments in another anatomical component. In the second part, we will demonstrate that the exploratory movements of each anatomical component contribute to individual differences - both across participants and across anatomical components - in the use of feedback from training and the transfer of competency during recalibration. This second part will focus on testing differences in exploratory movements between the different anatomical components and on testing the effects of these movements on the detection and sharing of information during transfer.

\section{Part 1: Experimental evidence for functional equivalence}

Anatomical similarities and functional equivalence

Anatomical explanations of transfer hinge on similarities between anatomical components (limbs and muscles). For instance, van Hedel, Biedermann, Erni, and Dietz (2002) showed that a newly acquired locomotor skill for bipedal obstacle avoidance transferred only to specific muscle types (flexors). Transfer can be remarkably specific to the kinds of anatomical components: Anstis (1995) found that aftereffects from one-legged hopping (using mostly extensors) do not transfer to the unadapted leg.

Explanations of transfer based on functional equivalence focus on the similarity across perceptual subsystems exhibited in the relationship among anatomical components, rather than in any anatomical component. Learning studies in interlimb coordination have uncovered the dominance of spatial over muscular transfer in learning phase relations (Temprado \& Swinnen, 2005). The spatial structure of these phase relations serves to maintain a symmetrical potential function that may support movement globally throughout the body (Haken, Kelso, \& Bunz, 1985). Competency will transfer from one phase relation to competency with the symmetrical phase relation (Zanone \& Kelso, 1997). Furthermore, learning of new phase relations transfers from trained upper limbs to untrained lower limbs and vice versa (Kelso \& Zanone, 2002). Transfer here depends on a functionally defined phase relation irrespective of anatomical differences.

The role of functional equivalence in transfer may have strong foundations in the organization of the sensorimotor cortex. One implication of functional equivalence across anatomically disparate components has been that aspects of transfer may unfold at abstract subcortical and cortical levels (e.g., Fabri \& Polonara, 2008). Crucially, in contrast to the traditional notions of somatotopic organization of the sensorimotor cortex, it appears that, for some tasks, cortical activity respects a functional, rather than a somatotopic, organization (e.g., Sanes \& Donoghue, 2000). In this case, competency resulting from training fingers to maintain a given sequence or phase relations will be encoded into groups of neurons that are specific to the sequence or phase relation (i.e., function), rather than to the control of fingers (i.e., anatomy; Grafton, Hazeltine, \& Ivry, 1998). Recent theories of neuroplasticity recast the nervous system as a field of neurons that solves motor problems adaptively by reorganizing anatomical components to assume new functional roles (Kleim, Jones, \& Schallert, 2003). Hence, there are both behavioral and neurological reasons to consider functional equivalence as a viable basis for transfer even when conditions of anatomical equivalence are not met.

Dynamic touch

We sought to investigate functional equivalence for transfer in the experimental paradigm of dynamic touch (Carello \& Turvey, 2004; Turvey, 1996; Turvey \& Carello, 1995). Dynamic touch registers properties of manipulated objects through muscle and tendon deformations. That is, dynamic touch is a mode of perception defined functionally, rather than anatomically, as movements made so as to discern the properties of wielded objects. We adapted the dynamic touch paradigm to research transfer of perceptual competency. Transfer of calibration from hand to contralateral hand had already been found in dynamic touch by Withagen and Michaels (2004). Our aim was to elaborate this finding by providing an account of how functional equivalence might support transfer of competency across anatomically disparate components. Hence, we sought to compare competency across anatomically disparate limbs (i.e., hand and foot) in a dynamic touch task. 
Dynamic touch for perceiving length can be performed by either foot or hand alone, with no difference in accuracy or variability between effectors (Hajnal, Fonseca, Harrison, Kinsella-Shaw, \& Carello, 2007). Functional equivalence between upper and lower limbs was also empirically demonstrated in dynamic-touch tasks requiring selective attention (Hajnal, Fonseca, Kinsella-Shaw, Silva, Carello, \& Turvey, 2007): When asked to perceive partial or whole length of unseen rigid rods by wielding with hand or foot, participants exhibited performance and variability that did not differ between effectors.

In the present experiment, participants were asked to perceive the length of various unseen rigid rods by wielding with hand and foot. They were told that veridical feedback would be provided during the training phase of the experiment. However, feedback was scaled up by a factor of 1.5 (see Withagen \& Michaels, 2004). We reasoned that inflated feedback makes for a stronger distinction between effects of feedback and effects of perceptual drift due to repeated exposure to the same set of stimuli. It is important to keep in mind that studying transfer boils down to a test of recalibration, and not a test of accuracy. Veridical feedback is a more compelling manipulation when studying accuracy. However, it is crucial that the experimental method does not conflate accuracy with recalibration. Scaling up feedback allows a better test of training in this case and, subsequently, transfer of competency. Veridical feedback may support transfer, and there may be interesting interactions between accuracy and transfer, but the present scope of the article is limited to understanding the transfer across anatomically disparate components. ${ }^{1}$ In keeping with Withagen and Michaels, we chose to omit veridical feedback altogether and, instead, simply test the effect of inflated feedback against the control case of no feedback.

\footnotetext{
${ }^{1}$ The use of visual information is prone to individual differences (see Withagen \& van Wermeskerken, 2009, for an example specific to visual feedback in dynamic touch) and nonlinearities (e.g., Steingrimsson, 2009; Stevens, 1957), both of which are at odds with conventional significance testing for mean differences (Dixon, 2005; Green \& Luce, 1974; Stephen et al., 2010). It would be premature to compound, with two kinds of feedback, the variability due to multiple kinds of feedback before we have a better understanding of transfer in general (i.e., irrespective of accuracy). Analytical clarity in standard ordinary least-squares (OLS) regression method (e.g., repeated-measures analysis of variance [ANOVA]) for demonstrating transfer across anatomical differences should be challenging enough given the potential for Type II error incident to nonlinearity and individual differences (e.g., Molenaar, 2008; Stephen \& Arzamarski, 2009). Hence, systematically introducing a further source of nonlinearity and individual differences would risk masking real effects of transfer. We will, in fact, pursue more complex analysis respecting some amount of the individual differences and nonlinearity in the second part of the article, but our case may hopefully be more compelling if it can first be demonstrated in a more traditional, more conservative analysis, such as an ANOVA.
}

We predicted that different effectors would exhibit transfer of calibration in dynamic touch, suggesting an effect of functional equivalence in the face of anatomical disparity. We hypothesized that the change in perceptual competency induced by training one effector would have both a shortand a long-term influence on the system as a whole. More specifically, we predicted that training with feedback would affect performance of both the trained and untrained effectors in the short and long terms, respectively. The foregoing predictions concerning functional equivalence will be tested here in the first part of the article. Because we expected that anatomical disparity between hand and foot would introduce asymmetries in exploration (e.g., Galloway \& Thelen, 2004) and, so, asymmetries in information detection (e.g., Stephen \& Arzamarski, 2009; Stephen, Arzamarski, \& Michaels, 2010; van de Langenberg et al., 2006), we used motion capture to collect data on the exploratory movements on each trial. However, predictions concerning the motion capture data will be postponed until the second part of the article. It is important first to establish the presence of functional equivalence before pursuing any anatomical qualifications on functional equivalence.

\section{Method}

Participants Thirty-two undergraduate students at the University of Connecticut participated. They received credit as part of fulfilling requirements in an introductory psychology course. All of them signed an informed consent form approved by the Institutional Review Board of the University of Connecticut.

Materials Rods were made of different materials (wood, aluminum, and plastic) with different diameters. To ensure that improvement did not involve identification of individual rods, different rod sets were used in the training and testing phases. $^{2}$ Both the test set and the training set consisted of 10 rods, the former ranging from 40 to $80 \mathrm{~cm}$, the latter from 40 to $85 \mathrm{~cm}$. The test set included rods of various materials and diameters, whereas the training set consisted only of wooden

\footnotetext{
$\overline{{ }^{2} \text { A pilot study }}$ was conducted to test whether participants learned to identify rods only in a single range of lengths. Five participants practiced perception of rod length in the pretest with rods that spanned from 30 to $70 \mathrm{~cm}$. They were trained with veridical feedback with the same set of rods. A posttest followed including all the rods from the pretest and the training block, in addition to 10 rods ranging from 80 to $120 \mathrm{~cm}$. Results revealed that when the training and test phases contained stimuli from different ranges of length, transfer of calibration still occurred. There was a significant decrease in absolute error between the short-range rods used in the pretest $(M=25 \%, S D=$ $0.071)$ and the long-range rods used in the posttest $(M=16 \%, S D=$ $0.032), t(4)=2.378, p<.05$. It was concluded that transfer generalized beyond a single range of stimuli.
} 
rods of identical diameter. A $15-\mathrm{cm}$ tube handle of $4.2-\mathrm{cm}$ diameter was screwed onto each rod so that the end of the object was flush with the end of the handle. A 20-cm-long plastic tube of $5.1-\mathrm{cm}$ diameter secured to the participant's shoe with duct tape was used as a foot handle. The specifications of both rod sets are given in Table 1. As is evident from the table, the test set consisted of pairs of rods that had the same length but, sometimes, very different mass.

Design Formally, this study followed a 2 (test: Phase I, or "pretest"; Phase III, or "posttest") $\times 2$ (Phase II (i.e., "training") effector: foot, hand) $\times 2$ (test effector: Phase I and Phase III, foot; Phase I and Phase III, hand) $\times 2$ (Phase II feedback: inflated feedback, no feedback) mixed design. Phase II effector and Phase II feedback were betweensubjects variables.

In total, there were four groups of participants. Half of the participants used the foot during Phase II, and the other half used the hand. Within each of these effector groups, half received training via feedback during this phase, and half did not. Both the pretest and posttest included a block of wielding with the hand and a block of wielding with the foot. In the training trials (Phase II), participants wielded with either the right hand or the right foot and, if they were in the feedback condition, received feedback after each trial. Hence, in terms of 10-trial blocks across the experiment, the foot-trained partic- ipants wielded with hand and then foot in Phase I, foot and then again foot in Phase II, and foot and then hand in Phase III; the hand-trained participants wielded with foot and then hand in Phase I, hand and then again hand in Phase II, and hand and then foot in Phase III. Ordering of effectors was the same for the control participants, the only difference being the absence of feedback.

Participants in the control groups were run first. Two linear regressions quantified the relationship between perceived length $\left(L_{\mathrm{p}}\right)$ and actual length $\left(L_{\mathrm{a}}\right)$ from all the training block trials of control participants: one regression equation for hand wielding, one for foot wielding. Experimental participants received feedback not on $L_{\mathrm{a}}$ but on a multiple of $L_{\mathrm{p}}$. Just as scaling up feedback allows a test distinguishing transfer from mere practice judging veridical length (e.g., Withagen \& Michaels, 2004), basing feedback on $L_{\mathrm{p}}$ ensured that transfer to a different limb would emerge as the result of experience with training, rather than of simply practice in judging veridical length. This rescaling used the slopes from the linear regressions of $L_{\mathrm{p}}$ on $L_{\mathrm{a}}$. For each regression (i.e., for hand or for foot), the slope $\left(c_{\text {control }}\right)$ indicated the relationship between $L_{\mathrm{a}}$ and $L_{\mathrm{p}}$. We derived a scaled-up feedback $(F B)$ for each limb by multiplying the slope $\left(c_{\text {control }}\right)$ of the appropriate regression line by a factor of 1.5 :

$F B=\left(1.5 \times c_{\text {control }} \times L_{\mathrm{a}}\right)+c_{\text {intercept }}$.
Table 1 Specifications of objects used in the experiment. Note that the inertial properties of wooden objects reflect inhomogeneities of the material

\begin{tabular}{llllll}
\hline Material & Diameter $(\mathrm{cm})$ & $\mathrm{L}_{\mathrm{a}}(\mathrm{cm})$ & $\mathrm{I}_{1}\left(\mathrm{~g}^{*} \mathrm{~cm}^{2}\right)$ & $\mathrm{I}_{3}\left(\mathrm{~g}^{*} \mathrm{~cm}^{2}\right)$ & Mass $(\mathrm{g})$ \\
\hline \multicolumn{2}{l}{ Test Stimuli in Phase I and Phase III } & & & & \\
PVC pipe & 3.18 & 40 & 120,360 & 6,080 & 264 \\
PVC pipe & 1.27 & 40 & 81,019 & 11,625 & 274 \\
PVC pipe & 3.18 & 60 & 403,810 & 8,831 & 387 \\
Wood & 1.27 & 60 & 148,930 & 12,252 & 238 \\
PVC pipe & 3.18 & 70 & 652,290 & 10,163 & 454 \\
Wood & 1.27 & 70 & 196,310 & 12,623 & 229 \\
Aluminum & 1.27 & 75 & 522,150 & 14,870 & 440 \\
Wood & 1.27 & 75 & 231,000 & 12,775 & 232 \\
Wood & 1.27 & 80 & 310,060 & 12,910 & 264 \\
PVC pipe & 1.27 & 80 & 470,740 & 15,439 & 364 \\
Training Stimuli & in & & & & \\
Wood & 2.10 & 40 & 47,060 & 6,890 & 159 \\
Wood & 2.10 & 45 & 63,917 & 7,412 & 170 \\
Wood & 2.10 & 50 & 117,290 & 8,618 & 230 \\
Wood & 2.10 & 55 & 148,980 & 8,960 & 239 \\
Wood & 2.10 & 60 & 149,610 & 8,646 & 210 \\
Wood & 2.10 & 65 & 187,190 & 8,927 & 220 \\
Wood & 2.10 & 70 & 215,370 & 9,000 & 218 \\
Wood & 2.10 & 75 & 281,220 & 9,427 & 240 \\
Wood & 2.10 & 85 & 337,770 & 9,647 & 249 \\
Wood & 2.10 & 380,990 & 9,691 & 248 \\
\hline
\end{tabular}


That is, participants in the experimental condition were trained to a scaling up of the actual-to-perceived mapping of lengths by participants in the control condition.

In Phase I (pretest), each participant in the two experimental groups received 10 trials of wielding by the effector not to be trained and 10 trials of wielding by the effector to be trained. In Phase III (posttest), each participant in the two experimental groups received 10 trials of wielding by trained effector and 10 trials of wielding by untrained effector. Each of the 10 stimulus objects was presented once per block of 10 trials. The order of trial blocks was counterbalanced by test effector. Phase II (training with inflated $F B$ ) distinguished the two experimental groups: One group received 20 trials restricted to wielding by foot, and one group received 20 trials restricted to wielding by hand. With an optional 2-min break halfway through the experiment, a typical session lasted $1 \mathrm{~h}$.

Procedure A diagram of the experimental setup is shown in Fig. 1. On all trials, a screen occluded the wielded rod, and participants indicated the length of the rod by moving a marker up and down on a vertical pulley system to estimate how far they could reach with the rod. The grasped end corresponded to the zero point on the pulley apparatus. The participant was seated on a mattress on the ground. For hand trials, the right hand was placed in a horizontal armrest at $45^{\circ}$ from the frontoparallel plane. On every trial, the object was placed into the gloved hand vertically, so that the end of the handle was flush with the bottom of the hand. A glove was used to prevent participants from identifying the same object in case it was given on two consecutive trials. Participants were told to maintain a firm grip during wielding and to restrict exploratory rotations of the wrist. For foot trials, an additional plastic tube handle was attached to the bottom of the right shoe with duct tape. For protection, the footwear was wrapped in a plastic bag. On each trial, an object was slid into the tube handle so that the initial orientation of the object was vertical. The tube was positioned such that the end of the object was flush with the heel. All exploratory motions were to be restricted to rotations around the ankle, with the rest of the leg kept as still as possible. No bending of the knee or hip was allowed.

On each trial, the participant made a perceptual judgment by positioning the marker to the maximal distance that could be reached with the rod, with the proximal end of the rod at the zero point on the pulley apparatus. On the inflated feedback trials given to the experimental participants, once participants were satisfied with their marker placement, the experimenter moved the marker to the distance that corresponded to the length calculated from the regression formula above. This spatial extent was always larger than the real length of the object and constituted inflated feedback. The participant was told that this was the real length of the object, and he or she was given opportunity to continue wielding the occluded
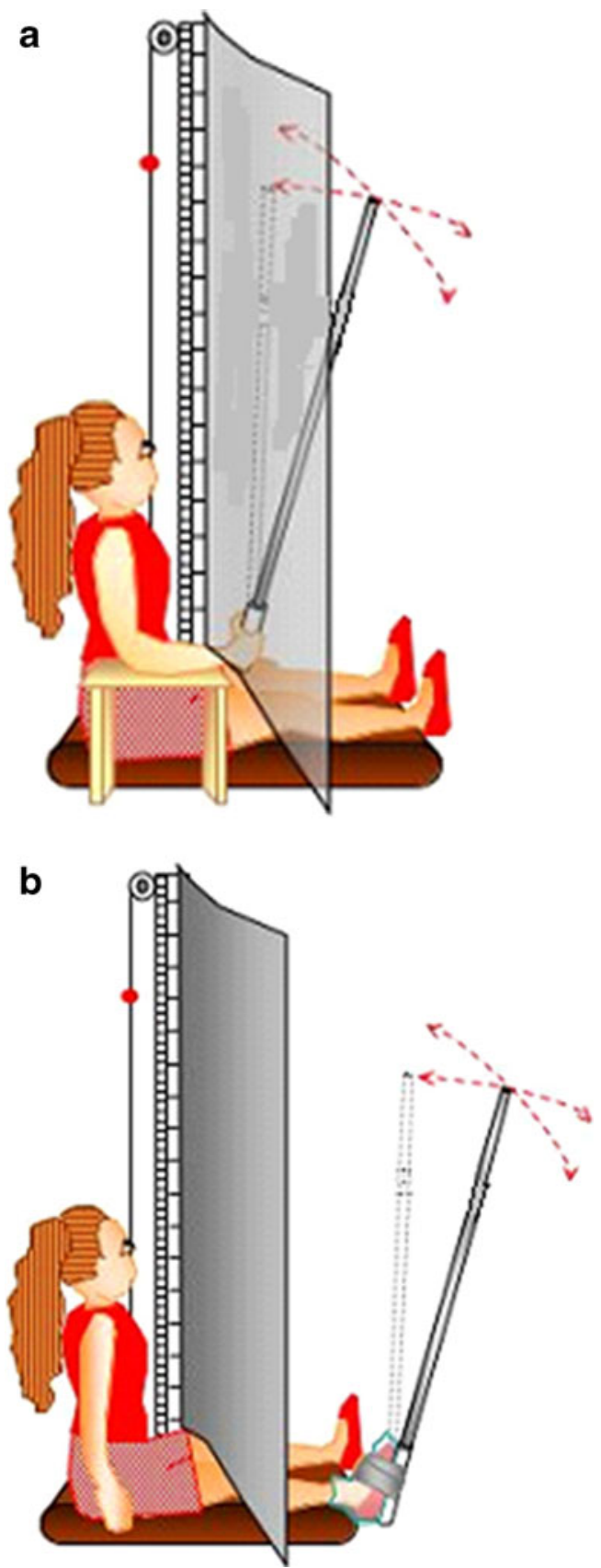

Fig. 1 The setup for hand wielding (a) and foot wielding (b) in Experiment 1

object while looking at the experimenter's indication of its alleged actual length.

Motion capture Three-dimensional motion capture of effector positions during wielding behaviors was recorded at $120 \mathrm{~Hz}$, using a magnetic tracking system (Polhemus Fastrak, Polhemus Corporation, Colchester, VT) and 6-D Research System software (Skill Technologies, Inc., Phoenix, AZ). Velcro was used to attach a motion capture marker to the wielding effector. For each trial, recording began on each trial 
when the participants began to wield the object, and recording ended when the participants were satisfied with their judgment. That is, recording did not include any wielding while the participant looked at the repositioned marker.

\section{Results}

Ratio of perceived length to actual length $\left(L_{p} / L_{a}\right)$ The ratio of $L_{\mathrm{p}}$ to $L_{\mathrm{a}}$ was used as a basic measure of performance. It is a dimensionless number that normalizes scores, allowing for easy comparisons between effectors. A 2 (test: Phase I, or pretest; Phase III, or posttest) $\times 2$ (Phase II effector: foot, hand) $\times 2$ (test effector: Phase I and Phase III, foot; Phase I and Phase III, hand) $\times 2$ (Phase II feedback: inflated feedback, no feedback) mixed analysis of variance (ANOVA) was conducted on $L_{\mathrm{p}} / L_{\mathrm{a}}$. There were a number of first- and second-order interactions, which were modified by a third-order interaction. We begin with the third-order, Phase II effector $\times$ test effector $\times$ test $\times$ Phase II feedback interaction, $F(1,28)=8.8, p<.006$. This interaction ispresented in Fig. 2. It shows that whereas foot training recalibrated the foot more than it did the hand, hand training recalibrated the hand and foot equally. This discrepancy percolated predictably through most of the other interactions. The significant test effector $\times$ test interaction, $F(1,28)=8.5$, $p<.007$, and the three-way test effector $\times$ test $\times$ Phase II feedback interaction, $F(1,28)=6.0, p<.02$, indicated that there was a wider gap between pre- and posttest in the case of foot judgments than in the case of hand judgments and that this gap was wider for foot judgments than for hand judgments as a result of feedback. The significant test effector $\times$ test $\times$ Phase II effector interaction, $F(1,28)=16.0$, $p<.001$, revealed that the pattern of results described by the test effector $\times$ test interaction was preserved but showed more recalibration when the foot was trained. The test

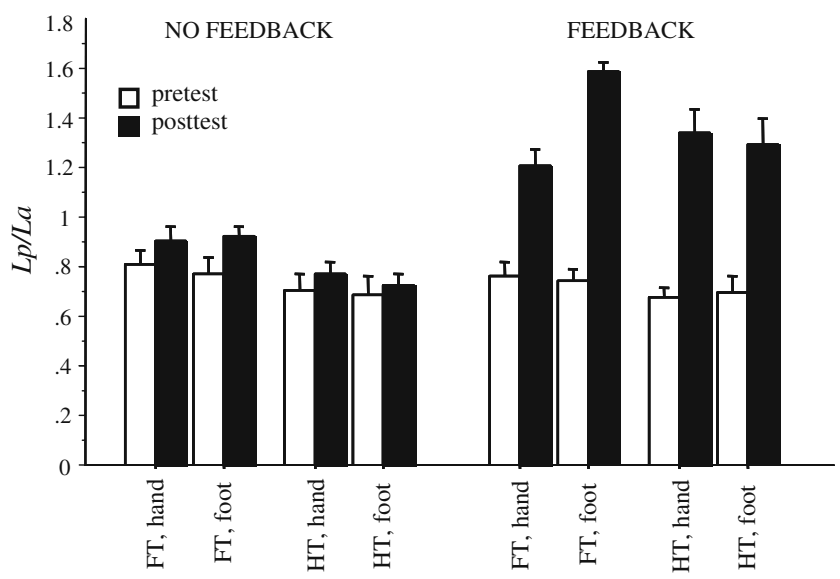

Fig. 2 Ratio of perceived rod length and actual length $\left(L_{\mathrm{p}} / L_{\mathrm{a}}\right)$. FT stands for foot training, HT for hand training. The error bars represent standard errors effector $\times$ Phase II feedback interaction was also significant, $F(1,28)=5.0, p<.03$, reflecting less recalibration in the control condition than in the feedback condition. The significant test effector $\times$ Phase II effector interaction, $F(1,28)=5.6, p<.02$, showed more recalibration with foot than with hand in the foot-training regime. The test $\times$ Phase II feedback interaction, $F(1,28)=109.9, p<.001$, indicated that feedback influenced both the trained and untrained effectors. Calibration transfer was confirmed by analyzing the degree to which the untrained effectors were recalibrated. Two test $\times$ Phase II feedback ANOVAs were conducted, one for hand training on perception by foot, and one for foot training on perception by hand. In both training regimes, the test $\times$ Phase II feedback interaction was significant $(p<.001)$, indicating that the untrained effector recalibrated as a result of feedback. This was a direct proof of recalibration and calibration transfer. Although the foot was recalibrated somewhat more than the hand, more transfer was observed from hand to foot than vice versa.

Reliability of judgments On average, feedback was scaled up by $45 \%$ and $42 \%$ from $L_{\mathrm{a}}$ for foot and hand, respectively. ${ }^{3}$ This rendered measures of accuracy with respect to actual length difficult to interpret. The reliability of judgments, defined as the average deviation of an individual's responses expressed as a percentage of that person's mean perceived length, was best suited to capture the consistency in the data:

reliability $=\frac{\sum_{i=1}^{N_{o}} \sum_{j=1}^{N_{\text {rep }}} \frac{\left|L p_{i j}-\overline{L p_{i}}\right|}{\overline{L p_{i}}}}{N_{o} \times N_{\text {rep }}} \times 100$,

where $L p_{i j}$ is the perceived length for object $i$ on the $j$ th trial, $\overline{L p_{i}}$ is the mean perceived length for object $i, N_{\mathrm{o}}$ is the number of objects used in the experiment, and $N_{\text {rep }}$ is the number of repetitions. ${ }^{4}$ The 2 (test effector: foot, hand) $\times 2$ (Phase II feedback: inflated feedback, no feedback) $\times 2$ (Phase II effector: foot, hand) mixed ANOVA on the difference between pretest and posttest reliability returned only a significant main effect of Phase II effector, $F(1,28)=5.7, p<.02$. That is, although both effectors were similarly reliable at the beginning of the experiment, reliability with foot training increased more than reliability with hand training. To offer a more detailed picture of the results, the two training regimes were reanalyzed by separate ANOVAs. A 2 (test effector: foot, hand) $\times 2$

\footnotetext{
${ }^{3}$ These feedback values were calculated for each stimulus object by plugging actual rod length into the rescaled regression equation. ${ }^{4}$ Strictly speaking, none of the objects were offered multiple times within a block. Since objects came in pairs of equal length, objects of the same length within a stimulus set were treated as multiple instances of the same length, and a mean was calculated.
} 
(Phase II feedback: inflated feedback, no feedback) mixed ANOVA was conducted on the difference between pretest and posttest reliability. The only significant effect was a main effect of Phase II feedback in the hand-training regime, $F(1,14)=5.9, p<.03$, suggesting that there was an overall increase of reliability in both the trained and untrained effectors from pretest to posttest as a result of feedback. No such increase was observed in the foottraining condition $(F<1)$.

\section{Discussion}

Overall, rescaling of $L_{\mathrm{p}} / L_{\mathrm{a}}$ showed evidence for the existence of transfer between different anatomical structures of the human body. That is, not only are the present results consistent with the existing findings that functional equivalence supports transfer in dynamic touch (Withagen \& Michaels, 2004), but also they suggest that functional equivalence is strong enough a substrate for transfer as to overcome considerable anatomical disparity, as between the hand and the foot.

Transfer of competency due to functional equivalence does not escape qualification by anatomical disparity. Perception of length was rescaled in both effectors as a result of feedback, but not by the same amount. More specifically, both the hand and the foot exhibited a rescaling of dynamic touch as a result of feedback irrespective of which effector was trained, but foot training facilitated less transfer to the hand than hand training did to the foot. We will highlight two major implications of this asymmetry across effectors.

In the first case, this asymmetry serves as new evidence that transfer by functional equivalence is deeply entangled in anatomical constraints of the motor system, further supporting the view that the "perceptual system" is unavoidably better thought of as a perception-action system (Gibson, 1979; Kugler \& Turvey, 1987; Thelen \& Smith, 1994). That is, in contrast to views presuming a fundamental divide between, first, the noisy degrees of freedom in the motor system and, second, a central executive processing mechanism to control them, the view of the organism in terms of a perception-action system suggests a broader distribution of function across all levels of biological activity. Under the framework of a perceptionaction system, function is less likely an abstract variable subject to strictly cognitive effects in the executive control mechanism. Even if function were an abstract regularity cognitively inferred from relatively noisy events at the motor periphery, its expression is impossible to divorce from the motor periphery. Hence, transfer can not be the central executive's simple reassignment of an abstract regularity to a different aspect of the motor periphery. Otherwise, transfer would have exhibited equivalent degrees of rescaling no matter which effector was trained. As it stands, the present results suggest that anatomical constraints influence not only the detection of haptic information, but also the later use of visual feedback for transfer. Transfer of competency may depend on functional equivalence, but function is not so easily disembodied from the motor system.

In the second case, the asymmetry between effectors raises further questions about what the relevant anatomical disparities might be and how they might help to explain the changes in transfer. The result of increased reliability only during hand training and the failure of reliability to increase overall both stand in contrast to the conventional wisdom that reliability will increase from pretest to posttest regardless of feedback (Gibson, 1969; Wagman, McBride, \& Trefzger, 2008). It may be that anatomical disparity between trained and tested effectors are to blame. The precedent for expecting reliability to increase overall regardless of feedback is based on a tradition of testing perceptual abilities within the same or anatomically similar effectors. Involving two anatomically disparate effectors, the present paradigm constitutes a marked departure from this tradition, and so the results do not necessarily contradict previous findings regarding reliability. The result simply raises the new challenge of understanding how the anatomical disparity influences transfer.

The simplest first conjecture might be that the asymmetry is due to each limb's having different capacities to detect information for dynamic touch. At first glance, it may seem surprising that the foot should exhibit stronger effects of transfer. After all, there is a well-documented superiority in sensory acuity (Kets, Van Leerdam, Van Brakel, Deville, \& Bertelsmann, 1996) and neurophysiological measures of sensitivity (Kennedy \& Inglis, 2002) of hands over feet. The coarser sensitivity of the foot may have to do with the tendency of foot movements to span a larger space (Lederman, Klatzky, Collins, \& Wardell, 1987). However, the traditional measures of sensitivity pertain to cutaneous touch, rather than to dynamic touch. The foot may actually be in a better position to detect a broader spectrum of information through dynamic touch. Not only are there more diverse synaptic inputs from the motor cortex in foot and leg muscles than in arm and hand muscles (Machii et al., 1999), but human infants will reach for objects with their feet before - and after - they learn to use their hands (Galloway $\&$ Thelen, 2004). Beyond infancy, the role of the feet in detecting useful haptic information may fade into the background (Bernstein, 1996; Turvey, 2007), but typically developing humans frequently rely on their feet to detect information specific to posture, gait, and even sitting (Ainscough-Potts, Morrissey, \& Critchley, 2006; Hirschfield, Thorsteinsdottir, \& Olsson, 1999; Kajita \& Tani, 1997; 
Kavounoudias, Roll, \& Roll, 2001; Perry, Santos, \& Patla, 2001). Grasping by the hand may seem more subtle than foot movements (Machii et al., 1999), but overall, upper limb movements expend less energy than do trunk and lower body movements that support posture and locomotion (Kumahara, Tanaka, \& Schutz, 2004). Although less salient, perhaps foot movements have an advantage over hand movements in making the most of transfer in haptic perception.

Despite the potentially endless list of differences between hand and foot, a good first place to begin looking for relevant differences is in exploratory movements. As was noted above and elsewhere, exploratory movements are crucial for detecting information (Gibson, 1966, 1979). Even within the same effector and the same task, a different exploratory mode can change the information detected from the same stimulus (Harrison, Hajnal, Lopresti-Goodman, Isenhower, \& Kinsella-Shaw, 2011; Stephen \& Arzamarski, 2009; van de Langenberg et al., 2006). Disparate anatomical constraints have immediate implications for changes in the available exploratory movements. A sensible strategy would thus be to examine the exploratory movements of the hand and the foot during the present task. Perhaps there are differences in these movements that might shed light onto why the foot appears more ready to take advantage of transfer in dynamic touch.

Having demonstrated the efficacy of functional equivalence for supporting transfer in the context of anatomical disparity, we will now revisit the same experiment as that reported above, and in the second part of the article, we will present an analysis of the motion-capture data that may provide some resolution for the present quandary. That is, in the second part of the article, we will seek to demonstrate that differences in transfer between hand and foot may be tied to differences in exploratory behaviors of the hand and the foot.

\section{Part 2: Investigating the role of exploratory movements in transfer}

Exploratory movements are inextricably related to the detection of information for perception. This relationship may be seen nowhere as clearly as in the sense of dynamic touch. Here, during the effortful wielding of an object, mechanical energy from the object's position and distribution of mass produces mechanical pressures on muscular tissues in the wielding limb. Registered by mechanoreceptors such as muscle spindles and Golgi tendon organs embedded in the muscles, these deformations carry information for judgments in dynamic touch (Carello \& Turvey, 2004). Additional evidence from nonhuman organisms, such as the wandering spider Cupiennius salei, also suggests that the patterning of pressures upon mechanoreceptors provides information about the geometrical extent of structure in the environment
(Barth, 2002). Essentially, haptic experience with environmental structure will produce neuromuscular fluctuations, and the structure of these fluctuations bear close relationship with the resulting perception of environmental structure. Our goal in this part of the article is to pursue the latter point with an analysis of exploratory movements, recorded during the experiment in the first part. The analysis of exploratory movements will aid in capturing the relationship between neuromuscular fluctuations and the limb differences in transfer of competency in dynamic touch.

Long-range temporal correlations in exploratory movements

It will be important, for present purposes, to recognize that neuromuscular fluctuations have memory. We intend the term memory not in the cognitive sense of personal recollection, but instead in the time series analysis sense of long-range temporal correlation. That is, neuromuscular fluctuations do not unfold strictly in the short term; the neuromuscular tissues are not a blank slate wiped clean after each action potential or muscle contraction. Rather, every change in these tissues is sensitive to the context set up by a history of physiological events, and every change, in turn, has repercussions for later physiological events (e.g., Carling, 2004; Chiel \& Beer, 1997; Feldman \& Latash, 2005; Hochachka, Darveau, Andrews, \& Suarez, 2003; Johnston $\&$ Edwards, 2002). If the neuromuscular fluctuations are to provide a foundation for the information detection in dynamic touch, it cannot be otherwise. The temporal patterning of wielding is crucial to the detection of information (Arzamarski et al., 2010; Stephen et al., 2010), and the neuromuscular tissues involved in wielding must share in this temporal patterning if they are, in the first place, to produce wielding movements and, in the second place, to detect information consequent to wielding. The long-range temporal correlations emerging within neuromuscular fluctuations must be crucial for the detection of information in dynamic touch.

One compelling expression of the memory in neuromuscular fluctuations is temporally fractal scaling (e.g., Werner, 2010). In temporally fractal scaling, root mean square (RMS) displacement (i.e., standard deviation of position in a trajectory integrated from individual displacements from an arbitrary origin; see Berg, 1993) increases according to a power law with time scale. The power law entails that RMS displacement increases quickly (i.e., according to a power of time scale), and due to the scale-invariant form of the power law, RMS displacement increases similarly quickly across all available time scales. Specifically, the power-law growth is substantially faster that what would be expected from the central limit theorem (CLT), according to which uncorrelated RMS displacement increases according to the square root of 
time (Shlesinger, Zaslavsky, \& Klafter, 1993). The power-law growth of fluctuations is evidence of long-range temporal correlations (Scafetta \& Grigolini, 2002; Viswanathan et al., 1996; Zanette, 1997). Temporally fractal scaling can be found in neuromuscular fluctuations from the coarser grain, such as posture (Duarte \& Zatsiorsky, 2001) and gait (Hausdorff et al., 1997), to a finer grain, such as breathing (Govindan, Wilson, Murphy, Russel, \& Lowery, 2007), heartbeats (Peng, Havlin, Stanley, \& Goldberger, 1995), and finger tapping (Lemoine, Torre, \& Delignières, 2006), to even finer grains, such as electric current in muscle (Gitter \& Czerniecki, 1995), neurotransmitter exocytosis (Lowen, Cash, Poo, \& Teich, 1997), ion-channel kinetics (Varanda, Liebovitch, Figueiroa, \& Nogueira, 2000), and interspike intervals in neuron firing (Das, Gebber, Barman, \& Lewis, 2003) - even independently of spinal influences (Orer, Das, Barman, \& Gebber, 2003). Temporally fractal scaling is a signature of memory throughout the neuromuscular apparatus available for dynamic touch.

The temporal fractality (i.e., the degree of temporally fractal scaling) may also be important for the efficacy of information detecting. Temporally fractal scaling is statistical evidence for the absence of a characteristic time scale. That is, within the sampling limits of a given measuring device, variability shows no sign of tapering off, no matter how coarse or how fine the scales chosen for analysis. We have already considered the definition of temporally fractal scaling on the time domain - that is, the power-law growth of RMS displacement over time. The absence of a characteristic time scale may be illustrated if we consider the evidence for temporally fractal scaling in the frequency domain: The spectral power of a temporally fractal time series will decay according to a power law with greater frequencies. Because of the scale-invariant form of a power law, measured temporally fractal time series will exhibit the participation of oscillations at all frequencies up to the sampling rate of the measuring device. Neuromuscular fluctuations may thus exploit oscillations on a wide (theoretically limitless) range of frequencies for the exploration of environmental structure and, in turn, the detection of information. That is, temporally fractal scaling allows exploration to be maximally flexible, responding even to details so fine or brief as to escape our conscious awareness. Sensitivity to events across such a wide variety of scales makes search behavior more thorough and effective (Bartumeus, Catalan, Fulco, Lyra, \& Viswanathan, 2002; Reynolds, 2010). Indeed, temporally fractal fluctuations in exploratory movements (by the hand and eye) facilitate the detection of information in perceptual tasks (Dixon, Stephen, Boncoddo, \& Anastas, 2010; Stephen \& Anastas, 2011; Stephen, Boncoddo, Magnuson, \& Dixon, 2009). Most relevant to the present article, temporally fractal fluctuations in wielding movements promote accuracy in judgments during dynamic touch by the hand (Stephen et al., 2010).
Beyond considerations of simply optimizing information detection, perhaps the more important point is that fractality may provide insights into the individual differences in information detection. Far from simply being a dichotomous property, fractality encompasses a continuous spectrum of long-range temporal correlations. As we have noted, temporally fractal scaling entails a power-law relationship between RMS displacement and time scale. That means that RMS displacement increases according to time scale raised to a scaling exponent $H$ (e.g., Kantelhardt et al., 2002; Mandelbrot, 1983; Scafetta, Moon, \& West, 2007). Temporally fractal scaling holds at least for the range $.5<H \leq 1$ (Kantelhardt et al., 2002; West, Geneston, \& Grigolini, 2008). Fractality is considered strongest for $H=1$. The trial-by-trial exponent $H$ for hand wielding in dynamic touch falls into the fractal range, and the closer $H$ was to 1 during exploration, the more accurate the following judgments became with training. Furthermore, trial-by-trial changes in this exponent $H$ also predicts trial-by-trial changes both in the use of inertial information for length judgments and in the use of visual feedback during training to rescale these judgments (Stephen et al., 2010). So, differences in the fractality of hand wielding predict differences in information detection for dynamic touch, both across time and across participants. Considering the longrange temporal correlations of temporally fractal scaling, this finding makes some sense: The quality of information detection for haptic perception should be related to the memory (i.e., temporal coherence) of the neuromuscular fluctuations involved in exploration.

Hypotheses regarding the effects of fractality on the transfer of competency

We now bring the foregoing insights to bear on the issue of transfer and, more specifically, on the questions raised in the first part of the article. Before, we had considered the potential for differences in exploratory movements to produce differences in transfer. As we have seen, the fractality of hand wielding predicts differences in information detection. Perhaps the differences in transfer across the two effectors (hand and foot) reflect differences in the fractality of the different effectors' wielding movements. Essentially, our experiment provides a ready foundation for clarifying further the role of fractality in perception. If the fractality of wielding behaviors does, in fact, indicate memory on the part of the neuromuscular fluctuations involved in dynamic touch, the fractality of wielding should predict how well training rescaled the judgments during the block immediately following training (i.e., for the trained limb) and during the final block (i.e., for the untrained limb). Stephen et al. (2010) had reported an effect of fractality on the use of feedback only during the training 
session, but we can now test for effects of fractality on the use of feedback not only by the trained limb after feedback has ended, but also by the untrained limb an entire block after feedback has ended. If all of these predictions were accurate, we would also expect a difference in fractality between the hand and the foot - at the very least, in the final block.

The direction of the expected difference between hand and foot wielding bears some further consideration. Stephen et al. (2010) found that fractality of wielding promotes accuracy, but they did so on the pretext of having given participants veridical feedback. Fractal wielding predicted use of inertial information and use of feedback for judgments, and the closer the scaling exponent $H$ was to 1 , the more accurate was the use of inertial information and feedback. Since the only feedback in our experiment was inflated, and not veridical, accurate use of the feedback would necessarily make our participants' responses less veridical. Still, accurate use of inertial information and accurate use of the inflated feedback would support transfer. So, in the present case, it makes more sense to predict that fractality should promote transfer. Hence, stronger fractality (i.e., scaling exponents closer to 1) should predict stronger transfer. In light of our findings in the first part of the article, because the foot showed stronger transfer than the hand, we would expect that the foot was more fractal (i.e., had scaling exponents closer to 1).

\section{Outline of hypotheses}

For clarity, we now outline the specific hypotheses that will structure our analysis. The first two hypotheses will be similar to those tested by Stephen et al. (2010), with the exception that they extend the same predictions to wielding by the foot, as well as by the hand. The last two hypotheses will move beyond the scope of Stephen et al. (2010) in addressing the relationship of fractality to transfer and differences in capacities of different effectors for detecting information under transfer.

Hypothesis 1 Our first prediction is that the fluctuations in wielding will be fractal, both for the hand and for the foot.

Hypothesis 2 Our second prediction is that the trial-by-trial degree of fractality, indicated by the estimated scaling exponent $H$, will help predict trial-by-trial changes in use of inertial information and also use of feedback during training.

Hypothesis 3 Our third prediction is that the trial-by-trial degree of fractality will predict the use of feedback both (1) for the trained limb after training has ended and (2) for the untrained limb in the transfer block.
Hypothesis 4 Our fourth prediction is that the fractality of the foot wielding will be greater (i.e., exhibiting trial-bytrial scaling exponents $H$ closer to 1 ) than that of the hand wielding during transfer. We anticipate this difference as a means of accounting for the asymmetry in transfer reported in the first part of this article. Note that this prediction is distinct from a simple expectation that $H$ will be closer to 1 for foot wielding than for hand wielding in general; an overall mean difference in $H$ between foot and hand wielding need not be either sufficient or necessary support for hypothesis 4 . Hypothesis 4 pertains specifically to an expected difference in $H$ during the transfer block.

\section{Analyses}

In order to test the four hypotheses enumerated above, we will need to draw on a variety of analyses. Testing hypothesis 1 will draw on three analyses: Detrended fluctuation analysis (DFA; Peng et al., 1994) will provide the primary estimates of the trial-by-trial fractal scaling exponents $H$, and dispersion analysis (Bassingthwaighte, Liebovitch, \& West, 1994; Holden, 2005) and autoregressive fractionally integrated moving average (ARFIMA) modeling (Granger \& Joyeux, 1980) will provide corroborating evidence of fractality. One shortcoming of DFA is that it may not be sufficient alone to distinguish between short-range and long-range temporal correlations, and corroborating evidence of long-range temporal correlations is necessary before the estimates of scaling exponent $H$ from DFA can be trusted to reflect fractality (Maraun, Rust, \& Timmer, 2004; Wagenmakers, Farrell, \& Ratcliff, 2004, 2005). Provided that a time series can be demonstrated to have long-range temporal correlations, DFA is one of the most reliable methods for estimating fractal scaling exponents (Oswiecimka, Kwapien, \& Drozdz, 2006; Peng et al., 1994; Torre, Delignières, \& Lemoine, 2007). Because DFA will be the primary analysis and because dispersion analysis and ARFIMA modeling will serve to corroborate evidence from DFA, only DFA will be described in this section, and descriptions of dispersion analysis and ARFIMA modeling may be found in Appendix 1 .

Detrended fluctuation analysis DFA begins with a time series $x(t)$ and integrates it to produce a random-walk trajectory $y(t)$, as follows:

$y(t)=\sum_{i=1}^{N} x(i)-\overline{x(t)}$,

where $\overline{x(t)}$ is the mean of $x(t)$. The individual values of $x(t)$ represent individual displacements, and $y(t)$ is a time series 
of positions relative to an arbitrary starting point. DFA calculates RMS displacement (i.e., standard deviation of positions) after removing local trends. Linear regressions $y_{n}(t)$ detrend nonoverlapping $n$-length windows of $y(t)$. Fluctuation $F(n)$ is calculated as average RMS error of these regressions for each $n$ :

$F(n)=\sqrt{(1 / N) \sum\left[y(t)-y_{n}(t)\right]^{2}}$

for $n<N / 4 .^{5}$ Similar to $S D$ in Eq. $1, F(n)$ increases as

$F(n) \sim n^{H}$.

Fractal time series generally exhibit scaling exponents $H$ in the range $.5<H \leq 1$, with stronger fractality yielding $H$ closer to 1 (e.g., Kiefer, Riley, Shockley, Villard, \& Van Orden, 2009). Logarithmic scaling of Eq. 3 yields

$\log F(n) \sim H \log n$.

The slope of $F(n)$ in double-log plots is taken to estimate $H$.

Growth curve modeling Testing hypotheses 2-4 will involve including the estimates of scaling exponents $H$ from DFA in growth curve modeling (GCM; Singer \& Willett, 2003) of the length judgments by dynamic touch. GCM is a multilevel modeling technique designed to test the effects of time-varying predictors on longitudinal data. Whereas standard ordinary least-squares (OLS) regression methods (e.g., ANOVA) assume homogeneous variance across participants and across time, GCM is a maximum likelihood (ML) regression method that fits random effects capturing individual differences, allowing for a more judicious estimate of fixed effects in the presence of heteroscedasticity. Because change over time may often manifest as heteroscedasticity (Molenaar, 2008), GCM is thus well equipped to model the changes in perceptual responses over time during training or transfer. GCM has already proven to be an effective way to examine haptic perceptual learning (Blau, Stephen, Carello, \& Turvey, 2009; Stephen \& Arzamarski, 2009; Stephen et al., 2010). We will use GCM to test the effects of time-varying predictors such as trial-by-trial scaling exponents $H$ on length judgments by dynamic touch, and this will help shed light on the effect of fractality on training and transfer.

Definition of predictors in GCM of length judgments In order to test the effects of fractality on length judgments in

\footnotetext{
${ }^{5}$ Alternately considering the scaling region with a smaller upper bound (i.e., $N / 10)$ provides a more conservative estimate of $H$ less prone to any instability in scaling resulting in the progressive scarcity of progressively larger windows (Hu, Ivanov, Chi, Carpena, \& Stanley, 2001).
}

dynamic touch, it will be necessary to incorporate a variety of predictors to properly account for the changes in length judgments. There is no value in testing the effect of fractality unless we control for all of the well-known factors contributing to responses in dynamic touch. First, we will want to control for practice in the task, represented by trial (trial number within each block, varying from 1 to 10) and block (block number, varying from 1 to 6 ). Second, we will want to control for the documented effects of the first and third inertial moments on judgments in dynamic touch (Arzamarski et al., 2010; Fitzpatrick, Carello, \& Turvey, 1994; Michaels, Arzamarski, Isenhower, \& Jacobs, 2008), represented by $I_{1}$ and $I_{3}$. Third, we will want to control for any effect of simple limb difference, represented by the dichotomous predictor hand (taking the value of 0 for each trial of foot wielding and 1 for each trial of hand wielding). Fourth, we will want to test effects of feedback, using three different dichotomous predictors: FB, PostFB, and Trans. FB will denote the effect of feedback during training; it will equal 0 for all trials except for those in blocks 3 and 4 (i.e., Phase II described in the experimental design), where it will equal 1 for the feedback condition. PostFB will denote the effect of having had feedback following training (i.e., during the block after feedback has ceased); it will equal 0 for all trials except for those in block 5 for the feedback condition. Trans will denote the effect of having had feedback following the change in effector (i.e., during the final block when dynamic touch is tested in the untrained effector); it will equal 0 for all trials except those in block 6 for the feedback condition. All of the foregoing predictors refer to the characteristics of the design. That is, they represent the contribution of experimental manipulation to length judgments in dynamic touch.

We introduce three different predictors to account for exploratory behaviors (i.e., the individual participants' contributions to their length judgments). Because we are primarily interested in fractality, we might simply include one predictor-namely, the trial-by-trial scaling exponent $H$ estimated by DFA. However, we would like to make an important distinction. Temporally fractal exploration should be distinguished from exploration that involves moving "more" or "more variably." Hence, in order to guard against the possible claim that any significant effects of trial-bytrial scaling exponent $H$ may be simply artifacts of moving "more" or "more variably," we introduce the predictors mean and $S D$ to denote the effects of the mean and the standard deviation of individual displacements in wielding movements.

In the following analyses, GCMs will proceed as the test of progressively higher-order interactions of the predictors listed above. In all cases, models will include all component 
terms (i.e., all lower-order interactions and main effects) for the most conservative test possible. Whereas OLS regression methods evaluate new effects with an $R$-squared statistic, the ML estimation requires that new effects be evaluated on the basis of how well the new effect reduces the $-2 \log$ likelihood ( $-2 \mathrm{LL}$ ) deviance statistic. The change in $-2 \mathrm{LL}$ will be evaluated as a chi-square test with degrees of freedom equal to the number of terms added to the model.

\section{Results}

Descriptives on wielding time series As was indicated in the first part of the article, motion capture during each trial in the task yielded a three-dimensional time series of foot or hand positions during wielding, with 120 samples per second. First, for each trial, we calculated the time series of individual displacements in wielding behavior by computing the Euclidean distance between each consecutive pair of threedimensional positions. For example, motion capture of $10 \mathrm{~s}$ of wielding would produce a three-dimensional position time series of 1,200 samples, and the corresponding time series of individual displacements would have 1,199 values. Thirty two participants completed 10 trials in each of six blocks of dynamic touch, generating a total of 1,920 (i.e., $32 \times 6 \times 10$ ) displacement time series. These individual-displacement time series had an average duration of 2,199 values $(S E=26.06)$, indicating that participants took, on average, $18.33 \mathrm{~s}$ [i.e., $(2199+1) / 120]$ to wield before deciding on a length judgment. An example individual-displacement time series is displayed in Fig. 3.

Testing hypothesis 1: Long-range temporal correlations in foot and hand wielding We submitted each time series to DFA. Estimates for trial-by-trial scaling exponents $H$ fell in the fractal range (i.e., $.5<H \leq 1$ ). Foot-wielding time series exhibited higher scaling exponents $(M=.85, S E=$ $.00)$ than did hand-wielding time series $(M=.79, S E=.00)$. As evidence that these fractal scaling exponents reflected correlations in the temporal sequence, randomly shuffled copies of the original time series were significantly lower scaling exponents for the foot $(M=.48, S E=.00)$ and for the hand $(M=.48, S E=.00)$, paired-samples $t(1919)=$ 82.61 and 66.68 , respectively, $p s<.0001$. Figures 4 and 5 depict example fluctuation functions $F(n) / n$ for foot and hand, respectively.

Testing hypothesis 1: Corroborating evidence of long-range temporal correlations Dispersion analysis yielded estimates of FD dimension consistent with long-range temporal correlations for both the foot $(M=1.25, S E=.00)$ and the hand $(M=1.29, S E=.00)$. ARFIMA modeling exhibited an improvement in fit above and beyond ARMA modeling. ${ }^{6}$ Summed weights for ARFIMA models were higher than those for ARMA models according to the Akaike information criterion (AIC) more often than by chance for both the foot and the hand, $\chi^{2}(1)=303.75$ and 507.50, respectively, $p s<.0001$. Similarly, summed weights for ARFIMA models were higher than those for ARMA models according to the Bayesian information criterion (BIC) more often than by chance for both the foot and the hand, $\chi^{2}(1)=$ 250.10 and 437.40, respectively, $p s<.0001$. For the AIC, $92.88 \%$ and $93.33 \%$ of the best-fitting ARFIMA models for foot and hand, respectively, estimated $d$ significantly greater than zero; for the BIC, $93.41 \%$ and $95.17 \%$ of the bestfitting ARFIMA models for foot and hand, respectively, estimated $d$ significantly greater than zero. Thus, ARFIMA modeling provided converging evidence of long-range temporal correlations in wielding.

As was noted above, the difference in $H$ between foot and hand might seem to provide tentative support for hypothesis 4 . That said, a mean difference across all trials and blocks in general need not hold specifically during the transfer block. Furthermore, until hypotheses 2 and 3 can be tested and until we have any evidence for the role of fractality in information detection, there may be no grounds to expect, let alone interpret, any difference in $H$ during the transfer block.

Testing hypothesis 2: Effects of trial-by-trial fractality on the use of inertial information Before arriving at a test of the crucial points of this hypothesis, there is a great deal of model building that must be done to ensure that effects of fractality might not be better accounted for by more likely and less novel predictors. Models will be reported in terms of their highest order interactions, but they will include all lower-order component effects as well (e.g., Stephen et al., 2010). Parsimony will not be the goal; rather, we seek to test whether fractality, a variable traditionally excluded from theoretical discourse, is worth including in later theoretical discourse at all. To that end, we were as conservative as possible and allowed many chances for alternate variables to absorb the predictive power. Furthermore, terms will be entered into the model in specific organizations that will allow test of the role of fractality in a variety of specific cases-for example, the role of fractality above and beyond limb differences during training, as distinct from the role of fractality above and beyond limb differences during transfer.

Because the progression of including terms will be somewhat tedious, we will include the details of this progression in Appendix 2. In all cases, modeling begins

\footnotetext{
${ }^{6}$ Augmented Dickey-Fuller tests for nonstationarity (i.e., "unit roots"; Banerjee, Dolado, Galbraith, \& Hendry, 1993) prior to ARMA and ARFIMA modeling rejected the hypothesis of nonstationarity for $99 \%$ of the time series, $p<.05$.
} 
Fig. 3 Example wielding time series from a single trial. The top three panels show each of three Euclidean dimensions of the wielding trajectory. The bottom panel shows the time series of Euclidean displacements between successive pairs of positions in the same trajectory. It is these Euclidean displacement time series that, for each participant on each trial, is submitted to DFA for estimation of the scaling exponent $H$
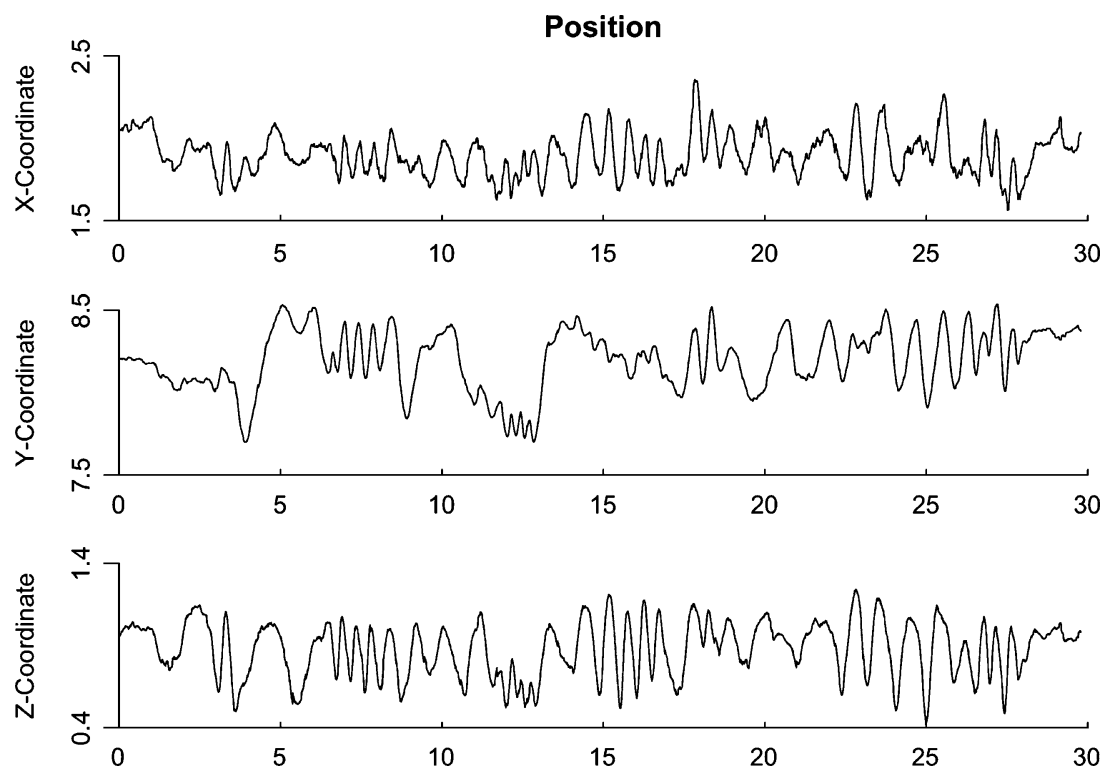

Displacement

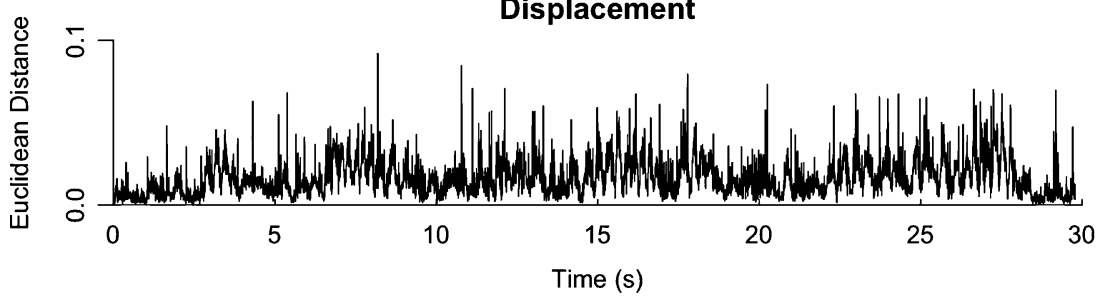

with an interaction of trial and block and proceeds by adding interactions specifying the part of the task during which a role of fractality is to be tested, the inertial information, the mean of fluctuations during wielding, and the standard deviation of fluctuations during wielding.

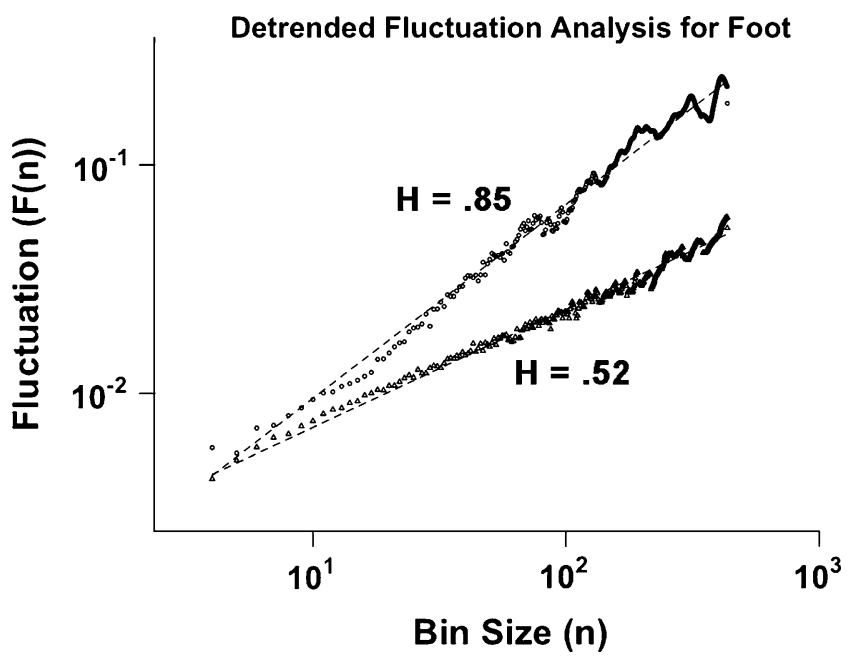

Fig. 4 Fluctuation function from DFA for a single example Euclidean displacement time series of foot wielding during an example trial by an example participant. Circles represent the fluctuation function for the original time series; triangles represent the fluctuation function for the shuffled copy of the time series
What will be reported in the main text is the result of the model at each step when fractality is entered.

Test of the effect of trial-by-trial fractality on the use of inertial information in general appeared in model 5 . Model 5 tested the $H^{*} S D^{*}$ mean ${ }^{*} \mathrm{I}_{1}{ }^{*}$ trial*block and $H^{*} S D^{*}$ mean ${ }^{*} \mathrm{I}_{3}$

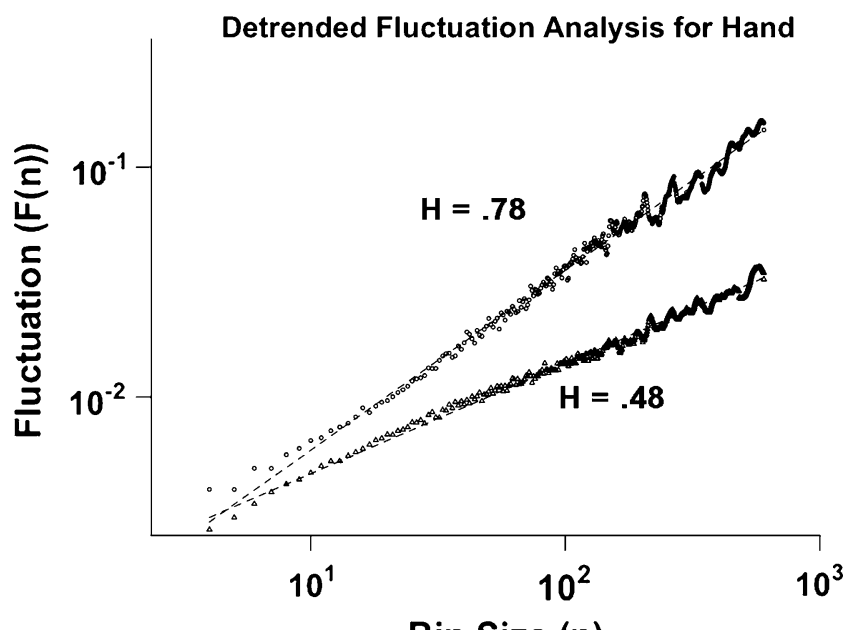

Fig. 5 Fluctuation function from DFA for a single example Euclidean displacement time series of hand wielding during an example trial by an example participant. Circles represent the fluctuation function for the original time series; triangles represent the fluctuation function for the shuffled copy of the time series 
*trial*block interactions, thus including the predictor $H$ as well as its interactions with all 47 terms in model 4 (i.e., adding 48 terms). The inclusion of $H$ terms improved prediction of length judgments, $\chi^{2}(48)=83.23, p<.01$. This result has twofold implications: First, it is consistent with existing evidence that the fractality of fluctuations in wielding predicted the use of inertial moments for generating length judgments in dynamic touch (Stephen et al., 2010); second, it extends that existing finding to a new limb, demonstrating that the effect of fractality may not be specific to wielding by the hand. See the first half of Table 2 for a summary of models leading up to this point.

Testing hypothesis 2: Effects of trial-by-trial fractality on the use of feedback during training Having tested the effect of fractality on the use of inertial moments for length judgments in dynamic touch, it was then necessary to test the effect of fractality on use of feedback during training. At this point, a crucial observation is that model 5 provided a model of length judgments general to all trials in the experimental procedure. That is, model 5 addressed only aspects of the experimental design that described the experience of all the participants, and it does not yet address those manipulations setting training condition apart from control condition. Subsequent modeling addressed these effects by including new sets of interactions of form similar to those in models 1-5 (see Appendix 2 for details).

Test of the effect of trial-by-trial fractality on the use of feedback during training appeared in model 10. Model 10 tested the hand $* H^{*} S D^{*}$ mean $* \mathrm{I}_{1} *$ trial*block, hand* $H^{*} S D$ ${ }^{*}$ mean ${ }^{*} \mathrm{I}_{3} *$ trial*block, $H^{*} S D^{*}$ mean*FB* $\mathrm{I}_{1} *$ trial*block, and $H^{*} S D^{*}$ mean $* \mathrm{FB}^{*} \mathrm{I}_{3} *$ trial*block interactions, thus including the interactions of $H$ with the 48 terms composing the FB interactions (i.e., adding 48 terms). The inclusion of these $H$ interactions improved prediction of length judgments, $\chi^{2}(48)=89.83, p<.001$. This result has twofold implications: First, it is consistent with existing evidence that the fractality of fluctuations in wielding predicted the use of inertial moments for generating length judgments in dynamic touch (Stephen et al., 2010); second, it extends that existing finding to a new limb, demonstrating that the effect of fractality may not be specific to wielding by the hand. ${ }^{7}$ In

\footnotetext{
${ }^{7}$ Additional modeling (omitted from the present article) demonstrated an improvement in prediction following inclusion of interactions of hand with all terms composing the FB interactions, except to note that including $H$ terms and hand terms improved prediction of length judgments irrespective of inclusion order. That is, as was noted above for model 6, effects of fractality did not exhaust effects of anatomical difference, but effects of fractality were also not simply artifacts of anatomical differences. Alternate modeling (also omitted from the present article) demonstrated similarly significant effects for estimates of $H$ from the more conservative scaling region specified by $\mathrm{Hu}$ et al. (2001). Those interested in further details may request them from the authors.
}

sum, fractality predicted changes in the use of feedback during training, above and beyond the effects of all other presently available predictors. See the second half of Table 2 for a summary of these models.

Testing hypothesis 3: Effects of trial-by-trial fractality on the use of feedback by the trained effector after training has ended Having tested for effects of fractality on the use of feedback during training, we could then test for the effects of fractality past the training blocks. That is, specifically, we could test for the effects of fractality in the trained effector during the block immediately following training (i.e., block 5). For this purpose, just as we had introduced new interactions with FB in previous modeling, we next introduced new interactions involving PostFB. The only catch is that, whereas feedback was given over two blocks (i.e., blocks 3 and 4), there was only one block with wielding by the trained effector after training ended (i.e., block 5). Hence, we could not articulate an interaction of PostFB (which equals 1 only for block 5 for participants in the training condition) and block. Modeling proceeded by introducing new interactions including PostFB where previous interactions had included block (see Appendix 2 for details; see Table 3).

Test of the effect of trial-by-trial fractality on the use of feedback by the trained effector after training appeared in model 14. Model 14 tested the hand* $H^{*} S D^{*}$ mean* $\mathrm{I}_{1} *$ trial *block, hand* $H^{*} S D^{*}$ mean* $\mathrm{I}_{3} *$ trial*block, $H^{*} S D^{*}$ mean * $\mathrm{FB}^{*} \mathrm{I}_{1} *$ trial*block, $H^{*} S D^{*}$ mean ${ }^{*} \mathrm{FB}^{*} \mathrm{I}_{3} *$ trial*block, $H^{*} S D^{*}$ mean ${ }^{*} \mathrm{I}_{1} *$ trial ${ }^{*}$ PostFB, and $H^{*} S D^{*}$ mean* $\mathrm{I}_{3} *$ trial *PostFB interactions, thus including the interactions of $H$ with the PostFB interactions (i.e., adding 24 terms). The inclusion of these $H$ terms improved prediction of length judgments, $\chi^{2}(24)=40.02, p<.05$, suggesting that the fractality of fluctuations in wielding predicted the use of feedback by the trained effector after training had ended. ${ }^{8}$ As during training, fractality predicted changes in use of feedback after training, above and beyond the effects of all other presently available predictors.

\footnotetext{
${ }^{8}$ As before in Footnote 7, additional modeling (omitted from the present article) demonstrated an improvement in prediction following inclusion of interactions of hand with all terms composing the FB interactions, except to note that including $H$ terms and hand terms improved prediction of length judgments irrespective of inclusion order. That is, as was noted above for model 6, effects of fractality did not exhaust effects of anatomical difference, but effects of fractality were also not simply artifacts of anatomical differences. This finding holds even when hand terms are included in earlier interactions, as noted in Footnote 7. If anything, inclusion of hand terms accentuates the improvement in prediction by subsequently including fractality. Alternate modeling (also omitted from the present article) demonstrated similar significant effects for estimates of $H$ from the more conservative scaling region specified by $\mathrm{Hu}$ et al. (2001). Those interested in further details may request them from the authors.
} 
Table 2 Summary of models testing hypothesis 2

\begin{tabular}{llll}
\hline Model & Predictors & Description of Included Effects & $p$ \\
\hline Effects on & use of inertial information & in & general \\
1 & Trial*Block & Experience in general & \\
2 & $\left(\mathrm{I}_{1}+\mathrm{I}_{3}\right) *$ Model 1 & Inertia in general & $<.0001$ \\
3 & Mean*Model 2 & Average individual displacement (ID) in general & $<.05$ \\
4 & SD*Model 3 & Variability of IDs in general & .13 \\
5 & $H^{*}$ Model 4 & Fractality of IDs in general & $<.01$ \\
Effects on the use of visual feedback during training & $<.0001$ \\
6 & Hand*Model 5 & Limb in general & $<.0001$ \\
7 & Model 6+FB*Model 2 & Experience with visual feedback during training & $<.05$ \\
8 & Model 6+FB*Model 3 & Average ID during training & $<.0001$ \\
9 & Model 6+FB*Model 4 & Variability of IDs during training & $<.001$ \\
10 & Model 6+FB*Model 5 & Fractality of IDs during training & \\
\hline
\end{tabular}

Testing hypothesis 3: Effects of trial-by-trial fractality on the use of feedback by the untrained effector during the transfer block Having tested for effects of fractality on the use of feedback by the trained effector after training, we could then test for the effects of fractality on the transfer of competency from the trained effector to the untrained effector. Whereas the effects on trained effector after training could be tested only in block 5 , the effects on the untrained effect could be tested only in block 6. Just as we had introduced interactions involving PostFB in the place of block, we adopted a similar strategy of including new interactions involving Trans in the place of block. Modeling proceeded by introducing new interactions including Trans where previous interactions had included block (see Appendix 2 for details).

Test of the effect of trial-by-trial fractality on the use of feedback by the untrained effector during transfer appeared in model 18. Model 18 tested the hand* $H^{*} S D^{*}$ mean* $I_{1} *$ trial * block, hand $* H^{*} S D^{*}$ mean* $\mathrm{I}_{3} *$ trial * block, $H^{*} S D^{*}$ mean $* \mathrm{FB}^{*} \mathrm{I}_{1} *$ trial $*$ block, $H^{*} S D^{*}$ mean $* \mathrm{FB}^{*} \mathrm{I}_{3} *$ trial $*$ block, $H^{*} S D^{*}$ mean ${ }^{*} \mathrm{FB}^{*} \mathrm{I}_{3} *$ trial * block, $H^{*} S D^{*}$ mean $* \mathrm{I}_{1} *$ trial*PostFB, $H^{*} S D^{*}$ mean* $\mathrm{I}_{3} *$ trial*PostFB, $H^{*} S D^{*}$ mean $* \mathrm{I}_{1} *$ trial*Trans, and $H^{*} S D^{*}$ mean ${ }^{*} \mathrm{I}_{3} *$ trial*Trans interactions, thus including the interactions of $H$ with the Trans interactions (i.e., adding 24 terms). The inclusion of these $H$ terms improved prediction of length judgments, $\chi^{2}(24)=40.71, p<.05$, suggesting that the fractality of fluctuations in wielding predicted the use of feedback by the trained effector after training had ended. ${ }^{9}$ As during training, fractality predicted changes in use of feedback by the untrained effector, above and beyond the effects of all other presently available predictors. That is, fractality predicted the degree of transfer in dynamic touch from one effector to another.

\footnotetext{
${ }^{9}$ Comments in Footnote 8 apply equally well at this stage of modeling. Once more, those interested in further details may request them from the authors.
}

Figures 6, 7, 8 and 9 depict the trial-by-trial length judgments for a representative sample of participants in each of the four conditions in the experiment, along with trial-bytrial prediction of length judgments from model 18. Let us recall that these four conditions differed both on the order of effectors used to wield (i.e., either hand, foot, foot, foot, foot, hand or foot, hand, hand, hand, hand, foot, for blocks 1-6, respectively, with the embedded order of blocks 1 and 2 and of blocks 5 and 6 counterbalanced, respectively) and on the presence or absence of inflated feedback during blocks 3 and 4. For the purpose of explaining the figures in the figure captions, we adopted the convention of referring to the conditions by the effector used during blocks 3 and 4 and by the presence or absence of feedback - hence, "foot control," "foot feedback," "hand control," and "hand feedback." In each figure, we chose to show trial-by-trial predictions from model 18 for the participants with the two best and the two worst pairwise correlations between actual judgment and predicted judgment.

Testing hypothesis 4: Difference in fractality between the effectors during the transfer block As was noted above in the test of hypothesis 1 , there was a mean difference in trialby-trial scaling exponent $H$ between the effectors when considered across the entire experiment. However, in light of the effects of fractality on transfer, we sought to determine whether the relative strength of transfer to the foot (i.e., as found in Part 1 of this article) reflected greater fractality of fluctuations in foot wielding specifically during the transfer block. To this end, we modeled trial-by-trial scaling exponent $H$ with another GCM. Modeling in this case differed from previous modeling in two ways. First, whereas previous modeling had used $H$ as a predictor in models of length judgment, we now treated $H$ as a dependent measure. Second, modeling now focused more on individual effects (i.e., the individual $B$ coefficients composing the regression model) and less on large sets of interactions. 
Table 3 Summary of models testing hypothesis 3

\begin{tabular}{|c|c|c|c|}
\hline Model & Predictors & Description of Included Effects & $p$ \\
\hline \multicolumn{4}{|c|}{ Effects on use of past feedback in block after training had ended (i.e., by trained limb) } \\
\hline 11 & Model $10+\operatorname{PostFB} *\left(\mathrm{I}_{1}+\mathrm{I}_{3}\right)^{*}$ trial & Past training for same limb & $<.0001$ \\
\hline 12 & Model $10+$ mean*PostFB* $\left(\mathrm{I}_{1}+\mathrm{I}_{3}\right) *$ trial & Average ID in posttraining block & .11 \\
\hline 13 & Model $10+S D^{*}$ mean*PostFB* $\left(\mathrm{I}_{1}+\mathrm{I}_{3}\right) *$ trial & Variability of IDs in posttraining block & .12 \\
\hline 14 & Model $10+H^{*} S D^{*}$ mean*PostFB* $\left(\mathrm{I}_{1}+\mathrm{I}_{3}\right) *$ trial & Fractality of IDs in posttraining block & $<.05$ \\
\hline \multicolumn{4}{|c|}{ Effects on the use of past feedback during transfer (i.e., by the untrained limb) } \\
\hline 15 & Model $14+$ Trans* $\left(I_{1}+I_{3}\right) *$ trial & Past training of other limb & $<.0001$ \\
\hline 16 & Model $14+$ mean*Trans* $\left(\mathrm{I}_{1}+\mathrm{I}_{3}\right) *$ trial & Average ID during transfer & .07 \\
\hline 17 & Model $14+S D *$ mean*Trans* $\left(\mathrm{I}_{1}+\mathrm{I}_{3}\right) *$ trial & Variability of IDs during transfer & $<.05$ \\
\hline 18 & Model $14+H^{*} S D^{*}$ mean*Trans* $\left(\mathrm{I}_{1}+\mathrm{I}_{3}\right) *$ trial & Fractality of IDs during training & $<.05$ \\
\hline
\end{tabular}

Model 19 modeled $H$ with the hand*Trans and trial*_ block interactions, as well as all constituent main effects (see Table 4). Trial*block represented terms controlling for overall drift in $H$ over the course of the experiment, irrespective of condition. The other interaction addressed the possibility of effector differences in $H$ during transfer. Model 19 yielded two effects on $H$. First, the positive effect of block $(B=.010, S E=.005, p=.07)$ indicated that fractality increased marginally in general. Second, the negative effect of hand $(B=-.061, S E=.015, p<.001)$ indicated that the fractality of fluctuations in foot wielding was consistently higher than that of fluctuations in hand wielding. The absence of a significant interaction of hand with Trans indicates that the mean difference in fractality between the effectors is not significantly changed in the transfer block. ${ }^{10}$

\section{General discussion}

In general, we had hypothesized that the changes in the temporal fractality of fluctuations in wielding would predict changes in length judgments during transfer and that this predictive relationship would improve prediction of differences in information detection across participants and across effectors. These expectations manifested more specifically as four hypotheses. Hypothesis 1 was that fluctuations in wielding would exhibit temporally fractal fluctuations in both effectors (i.e., hand and foot). Hypothesis 2 was that the temporal fractality of wielding would predict changes in the use of inertial information in general and changes in the use of

\footnotetext{
$\overline{{ }^{10} \text { Significant }}$ effect of hand and null effect of the hand*Trans interaction remain unchanged in alternate modeling (omitted from the present article) of estimates of $H$ from the more conservative scaling region specified by $\mathrm{Hu}$ et al. (2001).
}

visual feedback during training. Hypothesis 3 was that the temporal fractality of wielding would predict changes in the use of visual feedback during the block immediately following training (i.e., with wielding by the trained limb after feedback had ended) and changes in the use of visual feedback during the final block (i.e., with wielding by the untrained limb). Hypothesis 4 was that the temporal fractality of foot wielding would be stronger than that of hand wielding during the final block (i.e., the transfer block). In all cases, we found that the data were consistent with these hypotheses.

\section{Temporal fractality of wielding across limbs}

The finding of temporally fractal scaling demonstrated fractal fluctuations in exploratory movements by the hand and the foot. Fractality of fluctuations in responses by the hand has been widely demonstrated (Gilden, 2001; Kello, Beltz, Holden, \& Van Orden, 2007; Stephen \& Dixon, 2009; Torre et al., 2007; Treffner \& Kelso, 1999; Van Orden, Holden, \& Turvey, 2003), but the present finding extended the observation of temporal fractality to the responses by the foot. The finding of temporal fractality in foot wielding may be related to the temporal fractality in postural sway (Duarte \& Zatsiorsky, 2001). Indeed, it has often been argued that postural sway provides optical information for visual perception (Riccio \& Stoffregen, 1991; Stoffregen, Pagulayan, Bardy, \& Hettinger, 2000), and given the present evidence that fluctuations at the foot provide haptic information, it may be worth revisiting postural sway to determine whether its fractal fluctuations predict detection of optical information. Temporal fractality of fluctuations in foot wielding entailed that, similar to fluctuations in hand wielding (e.g., Stephen et al., 2010), fluctuation in foot wielding were long-range temporally correlated and exhibit a similarly fast (i.e., power-law) growth across multiple scales. The power-law growth of 
Fig. 6 Example plots of actual length judgments and predicted length judgments (from model

18) for the footexperimental condition. Predicted length judgments from model 18 correlated with actual judgments, $r \mathrm{~s}=.97$ (top left), .97 (top right), .95 (bottom left), and .93 (bottom right)
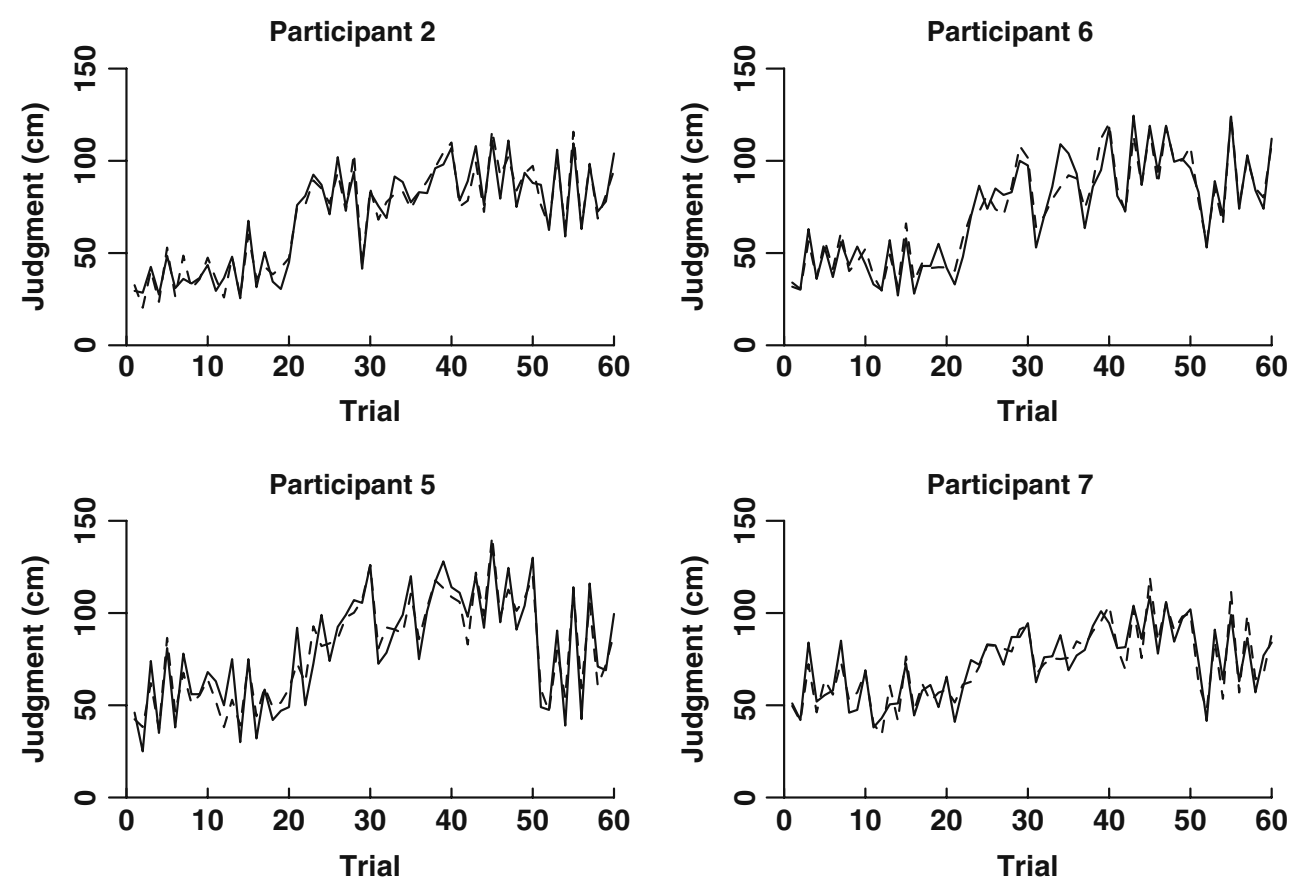

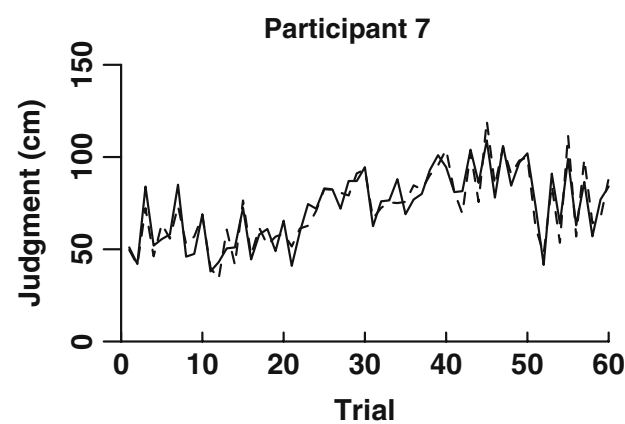

fluctuation - both in the hand and in the foot-suggested that exploratory movements are open to oscillations at all available frequencies.

Temporal fractality predicted use of inertial information and visual feedback

Previously, Stephen et al. (2010) had shown that the trialby-trial temporal fractality of fluctuations in hand wielding predicted changes in the use of inertial information during all blocks and visual feedback during training. The fact that, in the present study, both hand and foot wielding exhibited temporally fractal fluctuations allowed us to test whether the role of fractality in perceptual learning within limb would generalize to the case of transfer between two disparate limbs. The results provided evidence in favor of such a generalization of the role of fractality. The significant effects of including the trial-by-trial temporal fractal scaling exponent $H$ and its interactions in models 5 and 10 indicated that, as predicted in hypothesis 2 , the
Fig. 7 Example plots of actual length judgments and predicted length judgments (from model 18) for the footcontrol condition. Predicted length judgments from model 18 correlated with actual judgments, $r \mathrm{~s}=.90$ (top left), .89 (top right), .83 (bottom left), and .78 (bottom right)
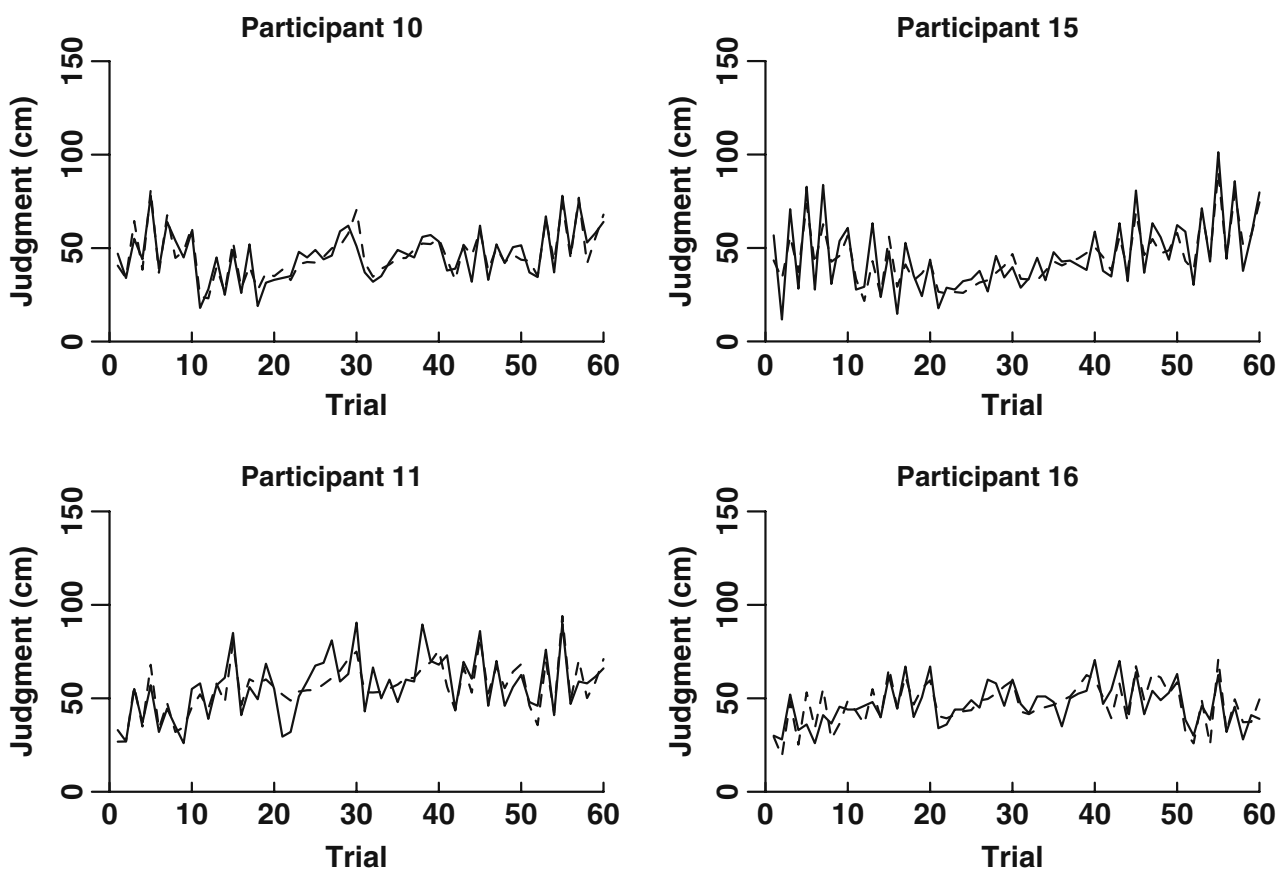
Fig. 8 Example plots of actual length judgments and predicted length judgments (from model 18) for the handexperimental condition. Predicted length judgments from model 18 correlated with actual judgments, $r \mathrm{~s}=.96$ (top left), .96 (top right), .89 (bottom left), and .71 (bottom right)
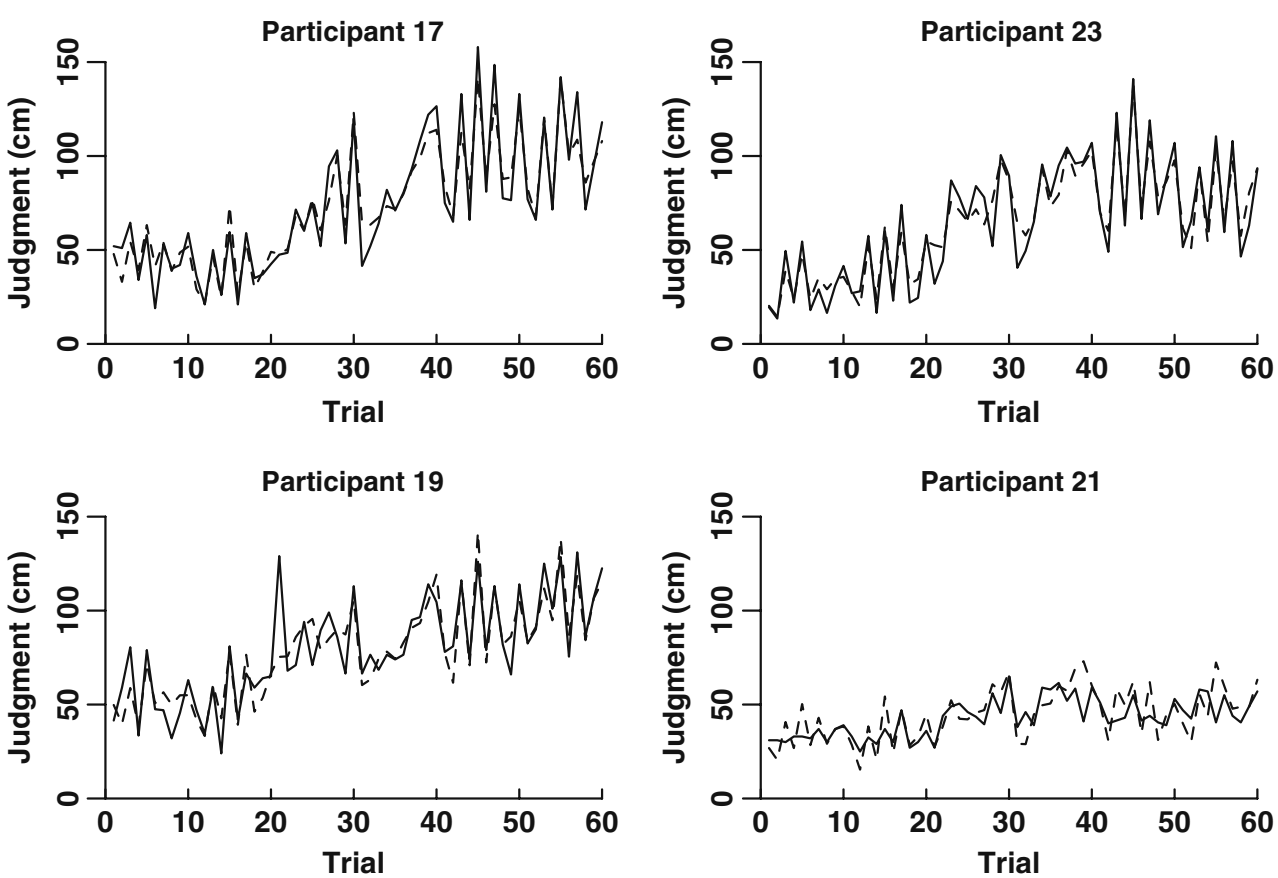

fractality of wielding, irrespective of limb, predicted changes in the use of inertial information in general and the use of visual feedback during training. These results replicated the findings of Stephen et al. (2010) for the effects of fractality in hand wielding and extended these findings to encompass fractality in foot wielding. In short, the present work suggested that temporal fractality may be a key attribute of exploratory movements irrespective of limb.
Temporal fractality predicted use of visual feedback after training had ended both for the trained and untrained limbs

The results of testing hypothesis 3 suggested the novel conclusion that the temporal fractality of fluctuations in wielding predicts how well the experience of having had visual feedback changed length judgments once training had ended. The findings by Stephen et al. (2010) and the results of testing hypothesis 2 in the present work addressed
Fig. 9 Example plots of actual length judgments and predicted length judgments (from model 18) for the handcontrol condition. Predicted length judgments from model 18 correlated with actual judgments, $r \mathrm{~s}=.94$ (top left), .91 (top right), .87 (bottom left), and .86 (bottomr ight)
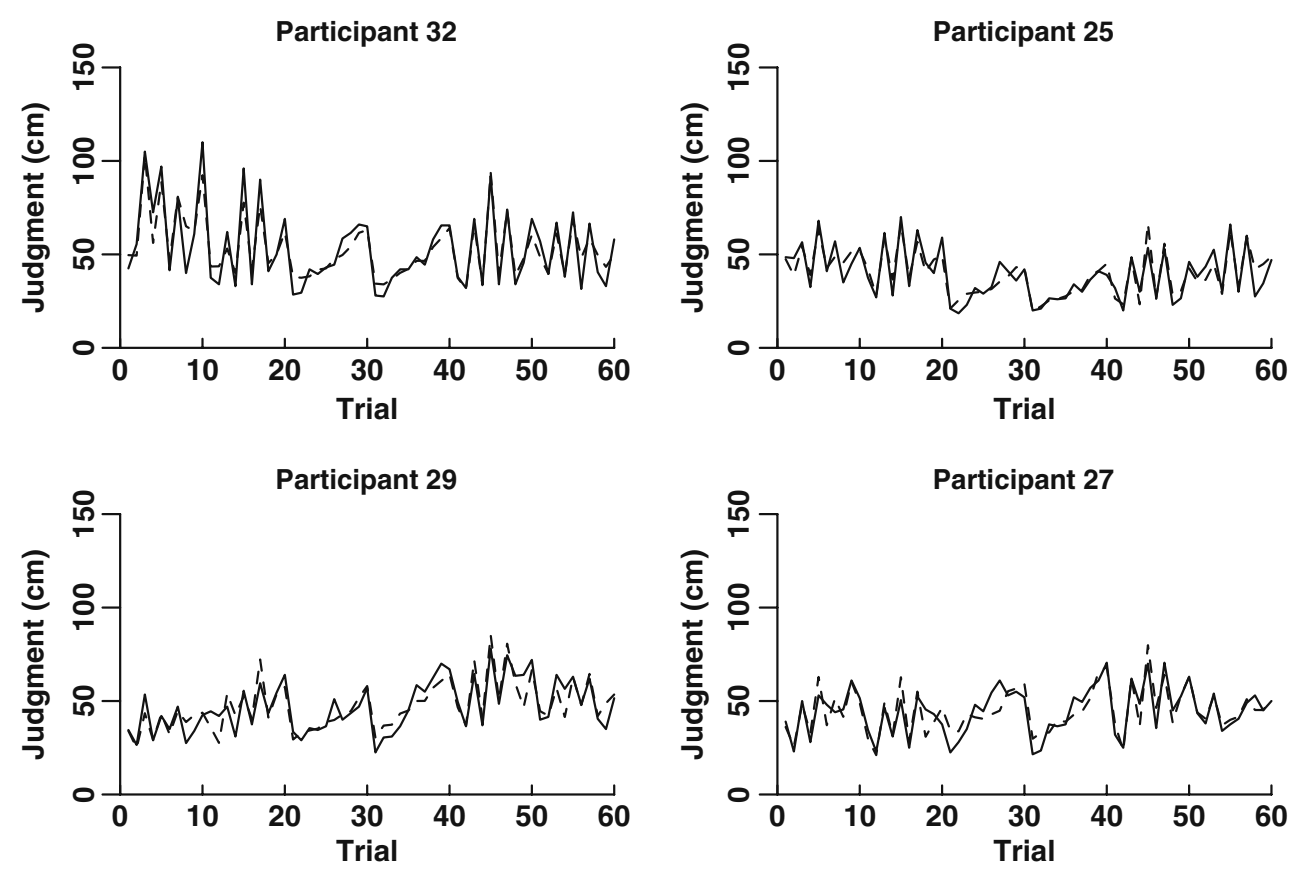
Table 4 Coefficients from model 19

\begin{tabular}{lcccc}
\hline Effect & $B$ & \multicolumn{1}{l}{$S E$} & $t$ & $p$ \\
\hline Intercept & .873 & .030 & 29.06 & $<.0001$ \\
Control effects & & & & \\
Block & .010 & .005 & 1.83 & .07 \\
Trial & .000 & .001 & 0.30 & .77 \\
Trial*block & .000 & .000 & 0.43 & .67 \\
Substantive effects & & & & \\
Hand & -.061 & .015 & -4.00 & $<.001$ \\
Trans & -.019 & .085 & -0.23 & .82 \\
Hand*Trans & .022 & .053 & 0.42 & .68 \\
\hline
\end{tabular}

only the effects of trial-by-trial fractality on use of information currently available on each trial. They did not speak to the possibility that trial-by-trial fractality predicted the use of previously available visual information from feedback once feedback was no longer available. Hypothesis 3 pressed upon the proposed role of long-range temporal correlations as an index of memory in neuromuscular fluctuations, predicting a relationship between the longrange temporal correlations of wielding after training had ended (i.e., after visual information for feedback was no longer available) and the success with which past experiences with feedback were woven together with current experiences in the posttest. The results provided evidence in favor of such a relationship. The significant effects of including the trial-bytrial temporal fractal scaling exponent $H$ and its interactions in models 14 and 18 indicated that, as predicted in hypothesis 3 , the fractality of wielding predicted use of visual feedback in the blocks after training ended. That is, fractality of wielding by both the trained limb and the untrained limb predicted changes in the effect of having had visual feedback on length judgments following training. In short, the present results suggested that temporal fractality reflected the memory of the perception-action system for its own history of information detection.

Temporal fractality differed across limbs in general and during transfer

Previous research had demonstrated that greater fractality (i.e., $H$ closer to 1) supported optimal use of available information (Stephen et al., 2010). In the present work, we predicted that the difference during the transfer block between foot and hand might reflect a difference in the fractality of hand wielding and of foot wielding. We had reported in the first part of the present article that transfer to the foot was stronger than transfer to the hand, and in this reanalysis, we predicted in hypothesis 4 that this limb difference might be partially attributable to a difference in temporal fractality. Because temporal fractality in wielding predicted the use of visual feedback (only provided during training) in the blocks after training had ended, it followed that a limb whose fluctuations exhibited a greater degree of fractality would be better able to make use of the information provided during training. The results provided evidence in favor of this explanation. The significant effect of hand and the nonsignificant effect of the interaction between hand and Trans in model 19 indicated that, as predicted in hypothesis 4 , the fractality of wielding was stronger in the foot than in the hand.

Fractality of wielding supported the transfer of competency between anatomically disparate limbs

We began this reanalysis with the goal of better understanding the asymmetry in transfer for different effectors. On the basis of previous research into the temporal structure of exploratory movements by the hand (Stephen et al., 2010), we proposed that the differences in use of information might be predicted from differences in temporal fractality of fluctuations in wielding. We have replicated the effects previously tested by Stephen et al. (2010) and expanded them not only to include a different limb, but also to demonstrate the role of temporal fractality in supporting the use of previously available information. Finally, we have shown that foot wielding exhibited stronger fractality than did hand wielding. We conclude that temporal fractality of exploratory movements plays a crucial role in the transfer of competency in dynamic touch.

Before we move on to the deeper theoretical interpretations of this finding, it is important to concede that fractality was by no means the only feature on which the limbs differed. We make no claims to having exhausted all possible sources of limb differences. Indeed, as was mentioned above in footnotes, alternate modeling not reported here demonstrated effects of hand on length judgments beyond effects of $H$ (i.e., fractality). The crucial aspect to note is that including hand effects prior to $H$ effects never prevented inclusion of $H$ effects from significantly improving model fit. That is, although temporal fractality may not constitute the only reason for differences in wielding by hand or by foot, temporal fractality is a specific feature of wielding behaviors that appeared to carry significant predictive weight in determining the use of information for the transfer of competency in dynamic touch.

Hyper-diffusivity and entailments of fractal fluctuations for information detection underlying perception

A great deal of interest and controversy has gone into attempting to infer the exact processes responsible for generating fractal scaling in perception-action and cogni- 
tion. Candidate processes include, at one explanatory extreme, the interaction-dominated dynamics of selforganizing systems in which multiplicative cascade processes weave together events across a wide variety of scales (e.g., Holden, Van Orden, \& Turvey, 2009; Ihlen \& Vereijken, 2010; Stephen \& Dixon, 2011; Stephen \& Mirman, 2010; Van Orden et al., 2003, 2005) and, at another explanatory extreme, the summed (i.e., not necessarily interactive) aggregation of arbitrarily many autoregressive processes (Ding, Chen, \& Kelso, 2002; Torre \& Wagenmakers, 2009; Wagenmakers et al., 2004, 2005; Ward, 2002). Because the variety in trial-by-trial (i.e., temporally local) fractality is sufficiently rich to support prediction of changes in consequent use of information, we might expect that wielding is multifractal (i.e., bearing multiple degrees of fractal scaling; e.g., Shlesinger et al., 1993) and, hence, indicative of interaction dominance (e.g., Ihlen \& Vereijken, 2010). We did not here present evidence adjudicating on this question, however, because we sought to address an alternate, often overlooked theoretical point about the relationship between fractality of exploratory movements - namely, that fractal scaling provides a window on energy flow involved in information detection underlying perception (Dixon, Holden, Mirman, \& Stephen, in press). Fractal scaling entails a markedly fast flow of energy, and the degree of fractality thus predicts the efficiency with which exploratory movements tap into the available energy distributions for perception. As exemplified by such classic physical examples as turbulence, fractal fluctuations are both symptomatic of quick energy flow (Shlesinger et al., 1993) and, perhaps more provocatively, capable of generating the quickest absorption and dispersion of injected energy, as in the case of fractal-generated turbulence (e.g., Seoud \& Vassilicos, 2007). Hence, fractal fluctuations in exploratory behaviors suggest a rapid absorption of ambient energy; changes in fractal fluctuations in exploratory behaviors thus bring about changes in this absorption.

The fluctuations in a motor signal reflect the flow of mechanical and electrical energy flowing through neuromuscular tissues. Motor fluctuations reflect the confluence of on-board potentials bound up in the physiology of the perception-action system and the potentials available in the environment (e.g., Kugler \& Turvey, 1987; Stephen et al., 2010). That is, a variety of physiological degrees of freedom composing the perception-action system are available for flushing ambient energy through the perception-action system. The consequent motor fluctuations thus provide a negative image of how these potentials diffuse over the course of exploratory movements. Provided the time series of individual displacements in any physiological signal follow the CLT (i.e., have a finite variance), the random-walk trajectory integrated from these individual displacements will cover distance (i.e., RMS displacement noted above) at a rate proportional to the flow of energy (Scafetta \& Grigolini, 2002; Shlesinger et al., 1993). Whereas ordinary mechanics generate randomwalk trajectories that generally exhibit a square-root growth of RMS displacement over time, temporal fractal scaling entails a faster (i.e., power-law) growth of RMS displacement in trajectory and, thus, entails faster diffusion-that is, hyperdiffusion. Differences in fractal scaling do not reflect differences in amount of movement (as shown by the effects of fractality above and beyond the effects of mean and $S D$ in foregoing models); rather, differences in fractality reflect differences in the temporal structure of fluctuations in the service of promoting energy flow.

The present research serves as the latest findings demonstrating that exploratory movements are fractal and the contention that the changes in fractality predict the efficacy of information detection. Previous research has documented such findings in the context of exploratory movements by the hand (Stephen \& Dixon, 2009; Stephen et al., 2010) or by the eye (Stephen \& Anastas, 2011; Stephen et al., 2009). The predictive power of fractality for capturing differences in information detection supports a view of perception-action as the evolving transaction of an organism with the patterned energy distribution in the environment (Gibson, 1966; Wagman \& Miller, 2003) without depending on a simple psychophysical correspondence (cf. Postman 1955; Stoffregen \& Bardy, 2001). That is, bare intensities of informational variables (e.g., moments of inertia) may well elicit various perceptual responses over time or across participants, but before plunging into the realm of cognitive processes for a means to explain this perceptual variety, fractal scaling of exploration is more plainly and more immediately available as a way to describe the role exploration can have in the variability in detection of information.

The present results extend previous work in that they demonstrate the generality of findings regarding temporal fractality not only to a new effector (i.e., the foot), but also across two different effectors within the same task. In short, the predictive power of fractality for capturing differences in information detection may be exported across effector in the same task. Fractal fluctuations thus may serve as a common currency through which the perception-action system can share information across multiple effectors or, conversely, through which an effector can draw upon the experiences of the perception-action system at large. Fractality of exploratory movements may thus be an important factor modulating the transfer of competency. It is known that different exploratory styles and constraints by the same limb can modulate the available information for dynamic touch (Harrison et al., 2011). Future research could investigate the deeper relation- 
ships between exploratory style and fractality of exploratory movements.

Transfer as a matter of function and fractality

Transfer occurs when information detected in one context influences the information detection in another, later context. Transfer depends on conditions of similarity between the earlier context and the later context (Blau et al., 2009). This article has offered a two-pronged approach to an understanding of transfer in the case of dynamic touch. We presented a task in which training in dynamic touch by one effector led to changes in dynamic touch by an anatomically disparate effector. What has emerged is a portrait of transfer as a subtle interplay of function and fractality.

First, we showed that functional equivalence is sufficient similarity for transfer of competency in dynamic touch. That is, transfer of training to one limb (e.g., the hand) is not limited to a homologous limb (e.g., the contralateral hand). Rather, recruiting disparate limbs for the same task can lead to transfer in spite of anatomical disparity. Function thus appears to play a large role in the detection of information by the perception-action system. ${ }^{11}$ Detected information is not strictly specific to the effector involved in detection; detected information is also specific to the function that occasions detection. However, our results in the first part of the article suggested that there was an asymmetry between effectors, such that dynamic touch by the foot reflected stronger transfer of competency from training of the hand than vice versa. So, the role of function in supporting transfer is not without qualification by anatomical disparity.

Second, we showed that temporal fractality of fluctuations in wielding predicted differences in information detection. Fractality predicted differences in information detection throughout the transfer paradigm: in general, during training, and after training across both limbs. Irrespective of any limb difference, temporal fractality appears to be a crucial feature of exploratory behaviors and to index the integration of information from neuromuscular experience over time. A significant difference in fractality across limbs held throughout the transfer paradigm: Wielding by the foot exhibited greater fractality than did wielding by the hand. We propose that the fractal advantage of the foot over the hand translated to an advantage in detecting information and, hence, in transferring competency for dynamic touch.

The portrait of transfer that emerges is one that melds functional equivalence and fractal differences. The first part of the article addressed the possibility that functional

\footnotetext{
${ }^{11}$ We intend "function" here as the goal-directed relationship between the organism and its environment irrespective of anatomical particulars; in this article, function is the perception of an object's extent purely by wielding.
}

equivalence was sufficient to effect transfer across anatomically disparate limbs. The latter part of the article addressed the possibility that differences in performance by anatomically disparate limbs were attributable to differences in fractality. In both cases, this article identifies complementary sides of the common ground needed for transfer to occur. On the one hand, function provides a taskdependent substrate across which the perception-action system can share information between its local subsystems (i.e., effectors). Although we have highlighted differences in fractality to explain differences in information detection, fractal fluctuations in general provide a substrate endogenous to the perception-action system. Functional equivalence may support transfer in the first place, and fractality may modulate the way that transfer unfolds through the heterogeneities of the perception-action system.

\section{Appendix 1}

\section{Dispersion analysis}

Dispersion analysis examines the change in standard deviation $D$ across sample means of nonoverlapping $n$-length subsets of the time series $x(t)$. $D(n)$ decays according to a power law,

$D(n) \sim n^{-c}$,

from which follows

$\log D(n) \sim-c \log n$.

The negative slope is used to calculate an estimate of fractal dimension (FD),

$F D=1-(-c)$.

A time series with long-range temporal correlations will exhibit $1.5>F D \geq 1$ (Aon, Cortassa, \& O'Rourke, 2006; Bassingthwaighte et al., 1994; Van Orden et al., 2003).

\section{ARFIMA modeling}

Conventionally, linear forecasting methods model time series in terms of short-range temporal correlations. When time series $x(t)$ is stationary, these methods attempt to model $x(t)$ in terms of autoregressive (AR) and moving average (MA) components. AR components describe the contribution of the $p$ previous values of $x(t)$ to its own current value:

$x(t)=\sum_{i=1}^{p} \phi_{i} x(t-1)+\varepsilon(t)$,

where $\phi_{i}$ is a coefficient denoting the effect of the $i$ th previous value on the current value of $x(t)$ and $\varepsilon(t)$ is a noise term. MA components describe the contribution of Gaussian 
fluctuations interacting with the $q$ previous values of $x(t)$ to its own current value:

$x(t)=\sum_{i=1}^{q} \theta_{i} \varepsilon(t-i)+\varepsilon(t)$,

where $\theta_{i}$ is a coefficient denoting the effect of the $i$ th previous Gaussian fluctuation. A model with both $p$ AR components and $q$ MA components is called an $\operatorname{ARMA}(p, q)$ model (Box \& Jenkins, 1970).

When $x(t)$ is nonstationary, it is said to have a unit root, meaning that it is the first-order integration of a stationary noise. Because AR and MA modeling presupposes stationarity, one strategy is to take the difference $x(t)-x(t-1)$ of the time series and to fit the (stationary) differences with AR and MA components. Hence, the linear model for time series with a unit root is an autoregressive integrated moving average (ARIMA) model that fits AR and MA terms to $x(t)-x(t-1)$, the differences of $x(t)$. In addition to $p$ AR components and $q$ MA components, ARIMA models have a $d$ parameter indicating the number of differencings of $x(t)$ required before $p$ AR and $q$ MA components can be fit to the data (Box \& Jenkins, 1970). These models are denoted as $\operatorname{ARIMA}(p, d, q)$.

ARFIMA modeling is a variation on ARIMA modeling. When the parameter $d$ is set to fractional values, the model exhibits long-range temporal correlations: Each current value of $x(t)$ depends on previous values at all lags. When $-.5 \leq d<0$, the long-range temporal correlations are negative; when $.5 \geq d>0$, they are positive. ARFIMA modeling allows a test for longrange temporal correlations (i.e., significantly nonzero $|d| \leq .5$ for the I component) above and beyond shortrange temporal correlations (i.e., the $p$ AR and $q \mathrm{MA}$ components; Torre et al., 2007; Wagenmakers et al., 2004, 2005). Essentially, this method amounts to fitting nine $\operatorname{ARMA}(p, q)$ models and nine $\operatorname{ARFIMA}(p, d, q)$ models for which $p$ and $q$ can vary among 0,1 , and 2 and letting the ARFIMA models estimate the optimized value of $d$. The best-fitting class of models for a given time series $x(t)$ is chosen according to either the AIC or the BIC, defined for the $i$ th model as

$$
\begin{aligned}
& A I C_{i}=-2 \log L_{i}+2 k_{i}, \\
& B I C_{i}=-2 \log L_{i}+k_{i} \log N_{i},
\end{aligned}
$$

where $\log L_{i}, k_{i}$, and $N_{i}$ are the $\log$-likelihood, degrees of freedom (i.e., $p+q+1$ for ARMA and $p+q+2$ for ARFIMA), and length of the time series, respectively, for the $i$ th model (Akaike, 1973; Schwarz, 1978). Each $i$ th model in a set of $m$ models is weighted as $w_{i}(A I C)$ or $w_{i}(B I C)$, where

$$
\begin{aligned}
& \Delta_{i}(A I C)=A I C_{i}-\min A I C, \\
& \Delta_{i}(B I C)=B I C_{i}-\min B I C, \\
& L_{i}(A I C) \sim \exp \left(-.5 \times \Delta_{i}(A I C)\right), \\
& L_{i}(B I C) \sim \exp \left(-.5 \times \Delta_{i}(B I C)\right), \\
& w_{i}(A I C)=\frac{L_{i}(A I C)}{\sum_{j=1}^{m} L_{j}(A I C)}, \\
& w_{i}(B I C)=\frac{L_{i}(B I C)}{\sum_{j=1}^{m} L_{j}(B I C)} .
\end{aligned}
$$

When a time series has long-range temporal correlations, the sum of weights for ARFIMA models will exceed the sum of weights for ARMA models, and the highestweighted ARFIMA model will have a significantly nonzero $|d| \leq .5$ (Torre et al., 2007). In general, adding parameters to any model should improve its fit. The main question is whether the added parameter, here $d$ added to the ARMA model in order to produce an ARFIMA, has a significant effect.

\section{Appendix 2}

Preliminary models for testing an effect of fractality on use of inertial information

Model 1 served as the most sparse model of length judgments that could be extended across the entire experimental procedure-namely, the trial*block interaction (and implicitly, the main effects of trial and block). Completely devoid itself of any experimentally relevant information, this model predicted length judgments purely as a function of continued experience with dynamic touch, and it served only as the foundation upon which further, more experimentally informative predictors can be tested. Model 1 allowed all subsequent reasoning to deal with the role of every other predictor as it supported changes in length judgment over time, as in the case of training and transfer.

Subsequent modeling proceeded as follows. Model 2 tested the $\mathrm{I}_{1} *$ trial*block and $\mathrm{I}_{3}{ }^{*}$ trial*block interactions, thus including inertial predictors $\mathrm{I}_{1}$ and $\mathrm{I}_{3}$, as well as their interactions with all 3 terms in Model 1 (i.e., adding 8 terms). The inclusion of $\mathrm{I}_{1}$ and $\mathrm{I}_{3}$ terms improved prediction of length judgments, $\chi^{2}(8)=1,415.26, p<.0001$, consistent with existing findings that inertial moments can predict length judgments in dynamic touch (Arzamarski et al., 2010; Fitzpatrick et al., 1994; Michaels et al., 2008). Model 3 
tested the mean ${ }^{*} \mathrm{I}_{1} *$ trial $*$ block and mean $* \mathrm{I}_{3} *$ trial*block interactions, thus including the predictor mean as well as its interactions with all 11 terms in model 2 (i.e., adding 12 terms). The inclusion of mean terms improved prediction of length judgments, $\chi^{2}(12)=25.42, p<.05$, suggesting that the average size of fluctuations in wielding predicted the use of inertial moments in generating length judgments in dynamic touch. Model 4 tested the $S D^{*}$ mean* ${ }_{1}{ }_{1}$ trial*block and $S D^{*}$ mean ${ }^{*} \mathrm{I}_{3} *$ trial*block interactions, thus including the predictor $S D$ as well as its interactions with all 23 terms in model 3 (i.e., adding 24 terms). The inclusion of $S D$ terms did not improve prediction of length judgments, $\chi^{2}(24)=32.04$, $p=.13$, suggesting that the variability of fluctuations in wielding did not, in general, play a role in the use of inertial moments for generating length judgments in dynamic touch.

Preliminary models for testing an effect of fractality on use of visual feedback during training

Before we could explicitly test for effects of fractality on use of feedback during training, it was important to control for difference of effector in general across all trials (i.e., for those effects composing model 5). Because we had already found a significant difference in the fractality between foot and hand wielding, we wanted to safeguard against confusing subsequent effects of fractality (i.e., on the use of feedback during training, after training, or during transfer) with effects of anatomical differences across all trials. Hence, we ran model 6 to incorporate the predictor hand into interactions in model 5 general to all trials. So, model 6 tested the hand ${ }^{*} H^{*} S D^{*}$ mean* ${ }_{1}{ }_{1}{ }^{*}$ trial ${ }^{*}$ block and hand* $H^{*} S D^{*}$ mean ${ }^{*} I_{3} *$ trial*block interactions, thus including the predictor hand as well as its interactions with all 95 terms in model 5 (i.e., adding 96 terms). The inclusion of hand terms improved prediction of length judgments, $\chi^{2}(96)=244.00, p<.0001$, suggesting that the limb differences contributing to differences in length judgments are not exhausted by fractality. It is noteworthy that the inclusion of $H$ terms improved prediction of length judgments even if hand terms were already included in the model (i.e., if model 5 included hand terms and model 6 included $H$ terms, instead of vice versa as reported), indicating that effects of fractality were not trivial artifacts of anatomical differences. Essentially, fractality predicted changes in use of inertial moments above and beyond the effects of all other presently available predictors.

Now, we proceeded from model 6 to test the effect of fractality on use of feedback during training. Model 7 tested the hand* $H^{*} S D^{*}$ mean ${ }^{*} \mathrm{I}_{1} *$ trial*block, hand* $H^{*} S D^{*}$ mean ${ }^{*} \mathrm{I}_{3}$ *trial*block, $\mathrm{FB}^{*} \mathrm{I}_{1} *$ trial*block, and $\mathrm{FB}^{*} \mathrm{I}_{3} *$ trial*block interactions, thus including the predictor FB as well as its interactions with all 11 terms from model 2 (i.e., adding 12 terms). The inclusion of FB improved prediction of length judgments, $\chi^{2}(12)=207.25, p<.0001$, consistent with existing evidence that feedback contributes to changes in relationship between inertial moments and length judgments in dynamic touch (Michaels et al., 2008; Stephen et al., 2010). Subsequent modeling enhanced these new FB interactions. Model 8 tested the hand ${ }^{*} H^{*} S D^{*}$ mean $* \mathrm{I}_{1} *$ trial *block, hand* $H^{*} S D^{*}$ mean* $\mathrm{I}_{3} *$ trial*block, mean*FB* $\mathrm{I}_{1}$ *trial*block, and mean ${ }^{*} \mathrm{FB}^{*} \mathrm{I}_{3} *$ trial ${ }^{*}$ block interactions, thus including the interactions of mean with the 12 terms composing the FB interactions (i.e., adding 12 terms). The inclusion of these mean interactions improved prediction of length judgments, $\chi^{2}(12)=23.69, p<.05$, suggesting that the average size of fluctuations in wielding predicted the use of feedback during training. Model 9 tested the hand* $H^{*} S D$ *mean* $\mathrm{I}_{1}$ *trial*block, hand ${ }^{*} H^{*} S D^{*}$ mean* $\mathrm{I}_{3} *$ trial*block, $S D *$ mean*FB* $\mathrm{I}_{1} *$ trial*block, and $S D^{*}$ mean ${ }^{*} \mathrm{FB}^{*} \mathrm{I}_{3} *$ trial *block interactions, thus including the interactions of $S D$ with the 24 terms composing the FB interactions (i.e., adding 24 terms). The inclusion of these $S D$ interactions improved prediction of length judgments, $\chi^{2}(24)=59.27, p<.0001$, suggesting that the variability of fluctuations in wielding predicted the use of feedback during training.

Preliminary models for testing an effect of fractality on use of feedback by the trained effector after training had ended

Thus, Model 11 tested the hand* $H^{*} S D^{*}$ mean $\mathrm{I}_{1} *$ trial*block, hand* $H^{*} S D^{*}$ mean $\mathrm{I}_{3} *$ trial*block, $H^{*} S D^{*}$ mean*FB* $\mathrm{I}_{1}$ *trial*block, $H^{*} S D^{*}$ mean* $\mathrm{FB}^{*} \mathrm{I}_{3} *$ trial*block, $\mathrm{I}_{1} *$ trial *PostFB, and $\mathrm{I}_{3}{ }^{*}$ trial*PostFB interactions, thus including PostFB and its interactions with the five terms composing $\mathrm{I}_{1} *$ trial and $\mathrm{I}_{3}$ *trial (i.e., adding six terms). The inclusion of PostFB terms improved prediction of length judgments, $\chi^{2}(6)=106.69, p<.0001$, consistent with existing evidence that training has a lasting effect on the trained effector (Michaels et al., 2008; Wagman et al., 2008).

With the foregoing replacement of block effects with PostFB effects in model 11, subsequent modeling proceeded similarly as for tests of hypothesis 2 . Model 12 tested the hand ${ }^{*} H^{*} S D^{*}$ mean* $\mathrm{I}_{1} *$ trial*block, hand ${ }^{*} H^{*} S D^{*}$ mean ${ }^{*} \mathrm{I}_{3} *$ trial*block, $H^{*} S D^{*}$ mean*FB* $\mathrm{I}_{1} *$ trial*block, $H^{*} S D^{*}$ mean $* \mathrm{FB}^{*} \mathrm{I}_{3} *$ trial*block, mean* $\mathrm{I}_{1} *$ trial ${ }^{*} \mathrm{PostFB}$, and mean* $\mathrm{I}_{3} *$ trial*PostFB interactions, thus including the interactions of mean with the PostFB interactions (i.e., adding 6 terms). The inclusion of these mean terms did not improve prediction of length judgments, $\chi^{2}(6)=10.41, p=.11$, suggesting that the average size of fluctuations in wielding did not predict the use of feedback by the trained effector after training had ended. Model 13 tested the hand ${ }^{*} H^{*} S D^{*}$ mea$\mathrm{n} * \mathrm{I}_{1} *$ trial*block, hand $* H^{*} S D^{*}$ mean $* \mathrm{I}_{3} *$ trial*block, $H^{*} S D^{*}$ mean $* \mathrm{FB}^{*} \mathrm{I}_{1} *$ trial*block, $H^{*} S D^{*}$ mean $* \mathrm{FB}^{*} \mathrm{I}_{3} *$ trial *block, $S D^{*}$ mean* ${ }^{*}{ }_{1}$ trial*PostFB, and $S D^{*}$ mean* ${ }_{3}{ }^{*}$ trial 
* PostFB interactions, thus including the interactions of $S D$ with the PostFB interactions (i.e., adding 12 terms). The inclusion of these $S D$ terms did not improve prediction of length judgments, $\chi^{2}(12)=18.00, p=.12$, suggesting that the variability of fluctuations in wielding did not predict the use of feedback by the trained effector after training had ended.

Preliminary models for testing an effect of fractality on the use of feedback by the untrained effector during the transfer block

Thus, model 15 tested the hand ${ }^{*} H^{*} S D^{*}$ mean $* \mathrm{I}_{1} *$ trial*block, hand* $H^{*} S D^{*}$ mean ${ }^{*} \mathrm{I}_{3} *$ trial*block, $H^{*} S D^{*}$ mean*FB* $\mathrm{I}_{1}$ *trial*block, $H^{*} S D^{*}$ mean*FB* $\mathrm{I}_{3} *$ trial*block, $H^{*} S D$ *mean* $\mathrm{I}_{1} *$ trial*PostFB, $H^{*} S D^{*}$ mean $\mathrm{I}_{3} *$ trial*PostFB, $\mathrm{I}_{1}{ }^{*}$ trial*Trans, and $\mathrm{I}_{3} *$ trial ${ }^{*}$ Trans interactions, thus including Trans and its interactions with the five terms composing $\mathrm{I}_{1}$ *trial and $\mathrm{I}_{3} *$ trial (i.e., adding six terms). The inclusion of Trans terms improved prediction of length judgments, $\chi^{2}(6)=61.88, p<.0001$, consistent with the evidence in Part 1 of this article that training influenced dynamic touch by the untrained effector.

With the foregoing replacement of block effects with Trans effects in model 15 , subsequent modeling proceeded similarly as for tests of PostFB. Model 16 tested the hand* $H^{*} S D^{*}$ mea$\mathrm{n} * \mathrm{I}_{1} *$ trial*block, hand ${ }^{*} H^{*} S D^{*}$ mean $* \mathrm{I}_{3} *$ trial*block, $H^{*} S D^{*}$ mean*FB* $\mathrm{I}_{1} *$ trial*block, $H^{*} S D^{*}$ mean $* \mathrm{FB}^{*} \mathrm{I}_{3} *$ trial *block, $H^{*} S D^{*}$ mean*FB* $\mathrm{I}_{3} *$ trial*block, $H^{*} S D^{*}$ mean* $\mathrm{I}_{1}$ *trial*PostFB, $H^{*} S D^{*}$ mean $* \mathrm{I}_{3} *$ trial $^{*}$ PostFB, mean $* \mathrm{I}_{1}$ *trial*Trans, and mean* $\mathrm{I}_{3} *$ trial $*$ Trans interactions, thus including the interactions of mean with the Trans interactions (i.e., adding 6 terms). The inclusion of these mean terms did not improve prediction of length judgments, $\chi^{2}(6)=11.49$, $p=.07$, suggesting that the average size of fluctuations in wielding only marginally predicted the use of feedback by the untrained effector. Model 17 tested the hand* $H^{*} S D^{*}$ mean* $\mathrm{I}_{1} *$ trial*block, hand ${ }^{*} H^{*} S D^{*}$ mean $* \mathrm{I}_{3} *$ trial*block, $H^{*} S D^{*}$ mean*FB* $\mathrm{I}_{1} *$ trial*block, $H^{*} S D^{*}$ mean $* \mathrm{FB}^{*} \mathrm{I}_{3} *$ trial *block, $H^{*} S D^{*}$ mean*FB* $\mathrm{I}_{3} *$ trial*block, $H^{*} S D^{*}$ mean* $\mathrm{I}_{1}$ *trial*PostFB, $H^{*} S D^{*}$ mean* $\mathrm{I}_{3} *$ trial*PostFB, $S D^{*}$ mean ${ }^{*} \mathrm{I}_{1}$ *trial*Trans, and $S D^{*}$ mean* $\mathrm{I}_{3} *$ trial*Trans interactions, thus including the interactions of $S D$ with the Trans interactions (i.e., adding 12 terms). The inclusion of these $S D$ terms improved prediction of length judgments, $\chi^{2}(12)=21.00, p<$ .05 , suggesting that the variability of fluctuations in wielding predicted the use of feedback by the untrained effector.

\section{References}

Ainscough-Potts, A.-M., Morrissey, M. C., \& Critchley, D. (2006). The response of the transverse abdominis and internal oblique muscles to different postures. Manual Therapy, 11, 54-60.
Akaike, H. (1973). Information theory and an extension of the maximum likelihood principle. In B. N. Petrov \& F. Czáki (Eds.), Proceedings of the 2nd International Symposium on Information Theory (pp. 267-281). Budapest: Akademiai Kiadó.

Anstis, S. (1995). Aftereffects from jogging. Experimental Brain Research, 103, 476-478.

Aon, M. A., Cortassa, S., \& O'Rourke, B. (2006). The fundamental organization of cardiac mitochondria as a network of coupled oscillators. Biophysical Journal, 91, 4317-4327.

Arzamarski, R., Isenhower, R. W., Kay, B. A., Turvey, M. T., \& Michaels, C. F. (2010). Effects of intention and learning on attention to information in dynamic touch. Attention, Perception, \& Psychophysics, 78, 721-735.

Bannerjee, A., Dolado, J. J., Galbraith, J. W., \& Hendry, D. F. (1993). Cointegration, error correction, and the econometric analysis of non-stationary data. Oxford: Oxford University Press.

Barth, F. G. (2002). A spider's world: Senses and behavior. Berlin: Springer.

Bartumeus, F., Catalan, J., Fulco, U. L., Lyra, M. L., \& Viswanathan, G. M. (2002). Optimizing the encounter rate in biological interactions: Lévy versus Brownian strategies. Physical Review Letters, 88, 097901.

Bassingthwaighte, J. B., Liebovitch, L. S., \& West, B. J. (1994). Fractal physiology. New York: Oxford University Press.

Berg, H. C. (1993). Random walks in biology. Princeton, NJ: Princeton University Press.

Bernstein, N. A. (1996). On dexterity and its development (M. L. Latash, Trans.). In M. L. Latash \& M. T. Turvey (Eds.), Dexterity and its development (pp. 3-244). Mahwah, NJ: Erlbaum.

Blau, J. J. C., Stephen, D. G., Carello, C., \& Turvey, M. T. (2009). Prism adaptation of underhand throwing: Rotational inertia and the primary and latent aftereffect. Neuroscience Letters, 456, 54-58.

Box, G. E. P., \& Jenkins, G. M. (1970). Time series analysis, forecasting and control. San Francisco: Holden-Day.

Carello, C., \& Turvey, M. T. (2004). Physics and psychology of the muscle sense. Current Directions in Psychological Science, 13, 25-28.

Carling, D. (2004). The AMP-activated protein kinase cascade-A unifying system for energy control. Trends in Biochemical Sciences, 29, 18-24.

Chiel, H. J., \& Beer, R. D. (1997). The brain has a body: Adaptive behavior emerges from interactions of nervous system, body and environment. Trends in Neurosciences, 20, 553-557.

Das, M., Gebber, G. L., Barman, S. M., \& Lewis, C. D. (2003). Fractal properties of sympathetic nerve discharge. Journal of Neurophysiology, 89, 833-840.

Ding, M., Chen, Y., \& Kelso, J. A. S. (2002). Statistical analysis of timing errors. Brain and Cognition, 48, 98-106.

Dixon, J. A. (2005). Strong tests of developmental ordering hypothesis: Integrating evidence from the second moment. Child Development, 76, 1-23.

Dixon, J. A., Holden, J. G., Mirman, D., \& Stephen, D. G. (in press). Multifractal dynamics in the emergence of cognitive structure. Topics in Cognitive Science.

Dixon, J. A., Stephen, D. G., Boncoddo, R. A., \& Anastas, J. (2010). The self-organization of cognitive structure. In B. H. Ross (Ed.), The psychology of learning and motivation (Vol. 52, pp. 343384). Burlington: Academic Press.

Duarte, M., \& Zatsiorsky, V. M. (2001). Long-range correlations in human standing. Physics Letters A, 283, 124-128.

Fabri, M., \& Polonara, G. (2008). Role of corpus callosum in the interhemispheric transfer of somatosensory information: An fMRI study. In L. N. Bakker (Ed.), Brain mapping research developments (pp. 73-100). New York: Nova Science.

Feldman, A. G., \& Latash, M. L. (2005). Testing hypotheses and the advancement of science: Recent attempts to falsify the equilibrium point hypothesis. Experimental Brain Research, 161, 91-103. 
Fitzpatrick, P., Carello, C., \& Turvey, M. T. (1994). Eigenvalues of the inertia tensor and exteroception by the "muscular sense. Neuroscience, 60, 551-568.

Galloway, J. C., \& Thelen, E. (2004). Feet first: Object exploration in young infants. Infant Behavior \& Development, 27, 107-112.

Gibson, E. J. (1969). Principles of perceptual learning and development. New York: Appleton-Century-Crofts.

Gibson, J. J. (1966). The senses considered as perceptual systems. Boston: Houghton-Mifflin.

Gibson, J. J. (1979). The ecological approach to visual perception. Boston: Houghton-Mifflin.

Gilden, D. L. (2001). Cognitive emissions of $1 / f$ noise. Psychological Review, 108, 33-56.

Gitter, J. A., \& Czerniecki, M. J. (1995). Fractal analysis of the electromyographic interference pattern. Journal of Neuroscience Methods, 58, 103-108.

Govindan, R. B., Wilson, J. D., Murphy, P., Russel, W. A., \& Lowery, C. L. (2007). Scaling analysis of paces of fetal breathing, gross-body and extremity movements. Physica A, 386, 231-239.

Grafton, S. T., Hazeltine, E., \& Ivry, R. B. (1998). Abstract and effector-specific representations of motor sequences identified with PET. Journal of Neuroscience, 18, 9420-9428.

Granger, C. W. J., \& Joyeux, R. (1980). An introduction to longmemory models and fractional differencing. Journal of Time Series Analysis, 1, 15-29.

Green, D. M., \& Luce, R. D. (1974). Variability of magnitude estimates: A timing theory analysis. Perception \& Psychophysics, $15,291-300$

Hajnal, A., Fonseca, S., Harrison, S., Kinsella-Shaw, J., \& Carello, C. (2007a). Comparison of dynamic (effortful) touch by hand and foot. Journal of Motor Behavior, 39, 82-88.

Hajnal, A., Fonseca, S., Kinsella-Shaw, J., Silva, P., Carello, C., \& Turvey, M. T. (2007b). Haptic selective attention by foot and by hand. Neuroscience Letters, 419, 5-9.

Haken, H., Kelso, J. A. S., \& Bunz, H. (1985). A theoretical model of phase transitions in human hand movements. Biological Cybernetics, $51,347-356$.

Harrison, S. J., Hajnal, A., Lopresti-Goodman, S., Isenhower, R. W., \& Kinsella-Shaw, J. M. (2011). Perceiving action-relevant properties of tools through dynamic touch: Effects of mass distribution, exploration style, and intention. Journal of Experimental Psychology: Human Perception \& Performance, 37, 193-206.

Hausdorff, J. M., Mitchell, S. L., Firtion, R., Peng, C.-K., Cudkowicz, M. E., Wei, J. Y., (1997). Altered fractal dynamics of gait: Reduced stride-interval correlations with aging and Huntington's disease. Journal of Applied Physiology, 82, 262-269.

Hirschfield, H., Thorsteindottir, M., \& Olsson, E. (1999). Coordinated ground forces exerted by buttocks and feet are adequately programmed for weight transfer during sit-to-stand. Journal of Neurophysiology, 82, 3021-3029.

Hochachka, P. W., Darveau, C.-A., Andrews, R. D., \& Suarez, R. K. (2003). Allometric cascade: A model for resolving body mass effects on metabolism. Computational Biochemistry and Physiology, 134, 675-691.

Holden, J. G. (2005). Gauging the fractal dimension of cognitive performance. In M. A. Riley \& G. C. Van Orden (Eds.), Tutorials in contemporary nonlinear methods for the behavioral sciences (pp. 353-400).Retrieved February 20, 2005, from http://www. nsf.gov/sbe/bcs/pac/nmbs/nmbs.jsp

Holden, J. G., Van Orden, G. C., \& Turvey, M. T. (2009). Dispersion of response times reveals cognitive dynamics. Psychological Review, 116, 318-342.

Hu, K., Ivanov, P. C., Chi, Z., Carpena, P., \& Stanley, H. E. (2001). Effect of trends on detrended fluctuation analysis. Physical Review E, 64, 011114.
Ihlen, E. A. F., \& Vereijken, B. (2010). Interaction-dominant dynamics in human cognition: Beyond $1 / f^{\alpha}$ fluctuation. Journal of Experimental Psychology: General, 139, 436-463.

Johnston, T. D., \& Edwards, L. (2002). Genes, interaction, and the development of behavior. Psychological Review, 109, 26-34.

Kajita, S., \& Tani, K. (1997). Adaptive gait control of a biped robot based on realtime sensing of the ground profile. Autonomous Robots, 4, 297-305.

Kantelhardt, J. W., Zschiegner, S. A., Bunde, A., Havlin, S., Koscielny-Bunde, E., \& Stanley, H. E. (2002). Multifractal detrended fluctuation analysis of nonstationary time series. Physica A, 316, 87-114.

Kavounoudias, A., Roll, R., \& Roll, J.-P. (2001). Foot sole and ankle muscle inputs contribute jointly to human erect posture regulation. Journal of Physiology, 532, 869-878.

Kello, C. T., Beltz, B. C., Holden, J. G., \& Van Orden, G. C. (2007). The emergent cordination of cognitive function. Journal of Experimental Psychology: General, 136, 551-558.

Kelso, J. A. S., \& Zanone, P. G. (2002). Coordination dynamics of learning and transfer across different effector systems. Journal of Experimental Psychology: Human Perception \& Performance, 28, 776-797.

Kennedy, P. M., \& Inglis, J. T. (2002). Distribution and behaviour of glabrous cutaneous receptors in the human foot sole. Journal of Physiology, 538, 995-1002.

Kets, C. M., Van Leerdam, M. E., Van Brakel, W. H., Deville, W., \& Bertelsmann, F. W. (1996). Reference values for touch sensibility thresholds in healthy Nepalese volunteers. Leprosy Review, 67, $28-38$

Kiefer, A. W., Riley, M. A., Shockley, K., Villard, S., \& Van Orden, G. C. (2009). Walking changes the dynamics of cognitive estimates of time intervals. Journal of Experimental Psychology: Human Perception \& Performance, 35, 1532-1541.

Kleim, J. A., Jones, T. A., \& Schallert, T. (2003). Motor enrichment and the induction of plasticity before or after brain injury. Neurochemical Research, 28, 1757-1769.

Kugler, P. N., \& Turvey, M. T. (1987). Information, natural law, and the self-assembly of rhythmic movement. Hillsdale, NJ: Erlbaum.

Kumahara, H., Tanaka, H., \& Schutz, Y. (2004). Daily physical activity assessment: What is the importance of upper limb movements vs. whole body movements? International Journal of Obesity, 28, 1105-1110.

Lederman, S. J., Klatzky, R. L., Collins, A., \& Wardell, J. (1987). Exploring environments by hand or foot: Time-based heuristics for encoding distance in movement space. Journal of Experimental Psychology: Learning, Memory, and Cognition, 13, 606614.

Lemoine, L., Torre, K., \& Delignières, D. (2006). Testing for the presence of $1 / f$ noise in continuation tapping data. Canadian Journal of Experimental Psychology, 60, 247-257.

Lowen, S. B., Cash, S. S., Poo, M.-M., \& Teich, M. C. (1997). Quantal neurotransmitter secretion rate exhibits fractal behavior. Journal of Neuroscience, 17, 5666-5677.

Machii, K., Ugawa, Y., Terao, Y., Hanajima, R., Furubayashi, T., Mochizuki, H., (1999). Input-output organization of the foot motor area in humans. Clinical Neuropsychology, 110, 13151320 .

Mandelbrot, B. B. (1983). The fractal geometry of nature. San Francisco: Freeman.

Maraun, D., Rust, H., \& Timmer, J. (2004). Tempting longmemory-on the interpretation of DFA results. Nonlinear Processes in Geophysics, 11, 495-503.

Michaels, C. F., Arzamarski, R., Isenhower, R. W., \& Jacobs, D. M. (2008). Direct learning in dynamic touch. Journal of Experimental Psychology: Human Perception \& Performance, 34, 944957. 
Molenaar, P. C. M. (2008). On the implications of the classical ergodic theorems: Analysis of developmental processes has to focus on intra-individual variation. Developmental Psychobiology, 50, 6069.

Orer, H. S., Das, M., Barman, S. M., \& Gebber, G. L. (2003). Fractal activity generated independently by medullary sympathetic premotor and preganglionic sympathetic neurons. Journal of Neurophysiology, 90, 47-54.

Oswiecimka, P., Kwapien, J., \& Drozdz, S. (2006). Wavelet versus detrended fluctuation analysis of multifractal structures. Physical Review E, 74, 016103.

Peng, C.-K., Buldyrev, S. V., Havlin, S., Simons, M., Stanley, H. E., \& Goldberger, A. L. (1994). Mosaic organization of DNA nucleotides. Physical Review E, 49, 1685-1689.

Peng, C.-K., Havlin, S., Stanley, H. E., \& Goldberger, A. L. (1995). Quantification of scaling exponents and crossover phenomena in nonstationary heartbeat time series. Chaos, 5, 82-87.

Perry, S. D., Santos, L. C., \& Patla, A. E. (2001). Contribution of vision and cutaneous sensation to the control of centre of mass (COM) during gait termination. Brain Research, 913, 27-34.

Postman, L. (1955). Association theory and perceptual learning. Psychological Review, 62, 438-446.

Reynolds, A. M. (2010). Bridging the gulf between correlated random walks and Lévy walks: Autocorrelation as a source of Lévy walk movement patterns. Journal of the Royal Society: Interface, 7 , $1753-1758$.

Riccio, G. E., \& Stoffregen, T. A. (1991). An ecological theory of motion sickness and postural instability. Ecological Psychology, 3, 195-240.

Sanes, J. N., \& Donoghue, J. P. (2000). Plasticity and primary motor cortex. Annual Review of Neuroscience, 23, 393-415.

Scafetta, N., \& Grigolini, P. (2002). Scaling detection in time series: Diffusion entropy analysis. Physical Review E, 66, 036130.

Scafetta, N., Moon, R. E., \& West, B. J. (2007). Fractal response of physiological signals to stress conditions, environmental changes, and neurodegenerative diseases. Complexity, 12, 12-17.

Schwarz, G. E. (1978). Estimating the dimension of a model. Annals of Statistics, 6, 461-464.

Seoud, R. E., \& Vassilicos, J. C. (2007). Dissipation and decay of fractal-generated turbulence. Physics of Fluids, 19, 105108.

Shlesinger, M. F., Zaslavsky, G. M., \& Klafter, J. (1993). Strange kinetics. Nature, 363, 31-37.

Singer, J. D., \& Willett, J. B. (2003). Applied longitudinal data analysis: Modeling change and event occurrence. New York: Oxford University Press.

Steingrimsson, R. (2009). Evaluating a model of global psychophysical judgments for brightness: I. Behavioral properties of summations and productions. Attention, Perception, \& Psychophysics, 71, 1916-1930.

Stephen, D. G., \& Anastas, J. (2011). Fractal fluctuations in gaze speed visual search. Attention, Perception, \& Psychophysics, 73, 666-677

Stephen, D. G., \& Arzamarski, R. (2009). Self-training of dynamic touch: Striking improves judgment by wielding. Attention, Perception, \& Psychophysics, 71, 1717-1723.

Stephen, D. G., Arzamarski, R., \& Michaels, C. F. (2010). The role of fractality in perceptual learning: Exploration in dynamic touch. Journal of Experimental Psychology: Human Perception \& Performance, 36, 1161-1173.

Stephen, D. G., Boncoddo, R. A., Magnuson, J. S., \& Dixon, J. A. (2009). The dynamics of insight: Mathematical discovery as a phase transition. Memory \& Cognition, 37, 1132-1149.

Stephen, D. G., \& Dixon, J. A. (2009). The self-organization of insight: Entropy and power-laws in problem solving. Journal of Problem Solving, 2, 72-101.
Stephen, D. G., \& Dixon, J. A. (2011). Strong anticipation: Multifractal cascade dyamics modulate scaling in synchronization behaviors. Chaos, Solitons, \& Fractals, 44, 160-168.

Stephen, D. G., \& Mirman, D. (2010). Interactions dominate the dynamics of visual cognition. Cognition, 115, 154-165.

Stevens, S. S. (1957). On the psychophysical law. Psychological Review, 64, 153-181.

Stoffregen, T. A., \& Bardy, B. G. (2001). On specification and the senses. Behavioral \& Brain Sciences, 24, 195-261.

Stoffregen, T. A., Pagulayan, R. J., Bardy, B. G., \& Hettinger, L. J. (2000). Modulating postural control to facilitate visual performance. Human Movement Science, 19, 203-220.

Temprado, J. J., \& Swinnen, S. P. (2005). Dynamics of learning and transfer of muscular and spatial relative phase in bimanual: Evidence for abstract directional codes. Experimental Brain Research, 160, 180-188.

Thelen, E., \& Smith, L. B. (1994). A dynamic systems approach to the development of cognition and action. Cambridge, MA: MIT Press.

Torre, K., \& Wagenmakers, E.-J. (2009). Theories and models for 1/f noise in human movement science. Human Movement Science, $28,297-318$.

Torre, K., Delignières, D., \& Lemoine, L. (2007). Detection of longrange dependence and estimation of fractal exponents through ARFIMA modeling. British Journal of Mathematical and Statistical Psychology, 60, 85-106.

Treffner, P. J., \& Kelso, J. A. S. (1999). Dynamic encounters: Longmemory during functional stabilization. Ecological Psychology, 11, 103-137.

Turvey, M. T. (1996). Dynamic touch. American Psychologist, 51, $1134-1152$.

Turvey, M. T. (2007). Action and perception at the level of synergies. Human Movement Science, 26, 657-697.

Turvey, M. T., \& Carello, C. (1995). Dynamic touch. In W. Epstein \& S. Rogers (Eds.), Handbook of perception and cognition: Vol. 5. Perception of space and motion. New York: Academic Press.

van de Langenberg, R., Kingma, I., \& Beek, P. J. (2006). Mechanical invariants are implicated in dynamic touch as a function of salience in the stimulus flow. Journal of Experimental Psychology: Human Perception and Performance, 32, 1093-1106.

van Hedel, H. J. A., Biedermann, M., Erni, T., \& Dietz, V. (2002). Obstacle avoidance during human walking: Transfer of motor skill from one leg to the other. Journal of Physiology, 543, 709717.

Van Orden, G. C., Holden, J. G., \& Turvey, M. T. (2003). Selforganization of cognitive performance. Journal of Experimental Psychology: General, 132, 331-350.

Van Orden, G. C., Holden, J. G., \& Turvey, M. T. (2005). Human cognition and $1 / f$ scaling. Journal of Experimental Psychology: General, 134, 117-123.

Varanda, W. A., Liebovitch, L. S., Figueiroa, J. N., \& Nogueira, R. A. (2000). Hurst analysis applied to the study of single calciumactivated potassium channel kinetics. Journal of Theoretical Biology, 206, 343-353.

Viswanathan, G. M., Afanasyev, V., Buldyrev, S. V., Murphy, E. J., Prince, P. A., \& Stanley, H. E. (1996). Lévy flight search patterns of wandering albatrosses. Nature, 381, 413-415.

Wagenmakers, E.-J., Farrell, S., \& Ratcliff, R. (2004). Estimation and interpretation of $1 / f^{\alpha}$ noise in human cognition. Psychonomic Bulletin \& Review, 11, 579-615.

Wagenmakers, E.-J., Farrell, S., \& Ratcliff, R. (2005). Human cognition and a pile of sand: A discussion on serial correlations and self-organized criticality. Journal of Experimental Psychology: General, 134, 108-116. 
Wagman, J. B., McBride, D. M., \& Trefzger, A. J. (2008). Perceptual experience and post-test improvements in perceptual accuracy and consistency. Perception \& Psychophysics, 70, 1060-1067.

Wagman, J. B., \& Miller, D. B. (2003). Nested reciprocities: The organism-environment system in perception-action and development. Developmental Psychobiology, 42, 317-334.

Ward, L. (2002). Dynamical cognitive science. Cambridge, MA: MIT Press.

Werner, G. (2010). Fractals in the nervous system: Conceptual implications for theoretical neuroscience. Frontiers in Physiology, 1, 1-28.

West, B. J., Geneston, E. L., \& Grigolini, P. (2008). Maximizing information exchange between complex networks. Physics Reports, 468, 1-99.
Withagen, R., \& Michaels, C. F. (2004). Transfer of calibration in length perception by dynamic touch. Perception \& Psychophysics, 66, $1282-1292$.

Withagen, R., \& van Wermeskerken, M. (2009). Individual differences in learning to perceive length by dynamic touch: Evidence for variation in perceptual learning capacities. Attention, Perception, \& Psychophysics, 71, 64-75.

Zanette, D. H. (1997). Persistence in Lévy flight anomalous diffusion. Physical Review E, 55, 6632-6635.

Zanone, P. G., \& Kelso, J. A. S. (1997). Coordination dynamics of learning and transfer: Collective and component levels. Journal of Experimental Psychology: Human Perception and Performance, $23,1454-1480$. 